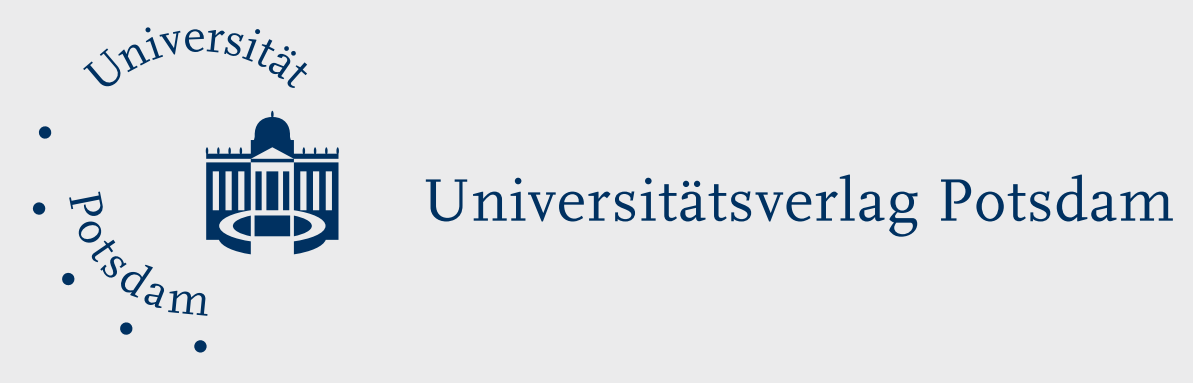

Justine Marienfeldt

\title{
Qualitätsmanagement in Nonprofit-Organisationen
}

Eine mikropolitische Analyse am Beispiel des Landesverbandes der Arbeiterwohlfahrt Berlin 

Schriftenreihe für Public und Nonprofit Management 

Schriftenreihe für Public und Nonprofit Management | 22

Justine Marienfeldt

\section{Qualitätsmanagement in Nonprofit-Organisationen}

Eine mikropolitische Analyse am Beispiel des Landesverbandes der Arbeiterwohlfahrt Berlin 
Bibliografische Information der Deutschen Nationalbibliothek

Die Deutsche Nationalbibliothek verzeichnet diese Publikation in der

Deutschen Nationalbibliografie; detaillierte bibliografische Daten sind

im Internet über http://dnb.dnb.de/ abrufbar.

Universitätsverlag Potsdam 2018

http://verlag.ub.uni-potsdam.de/

Am Neuen Palais 10, 14469 Potsdam

Tel.: +49 (0)3319772533 / Fax: 2292

E-Mail:verlag@uni-potsdam.de

Die Schriftenreihe für Public und Nonprofit Management wird herausgegeben vom Lehrstuhl für Public und Nonprofit Management der Wirtschafts- und Sozialwissenschaftlichen Fakultät der Universität Potsdam.

\section{ISSN (Online) 2190-4561}

Dieses Werk ist unter einem Creative Commons Lizenzvertrag lizenziert: Namensnennung - Weitergabe zu gleichen Bedingungen 4.0 International Um die Bedingungen der Lizenz einzusehen, folgen Sie bitte dem Hyperlink: http://creativecommons.org/licenses/by-sa/4.0/

Potsdam, Univ., Bachelorarbeit, 2018

Erstgutachter: Prof. Dr. Isabella Proeller Lehrstuhl für Public und Nonprofit Management

Zweitgutachter: Prof. Dr. John Siegel

Vertreter der Professur für Public und Nonprofit Management

Online veröffentlicht auf dem Publikationsserver der Universität Potsdam URN urn:nbn:de:kobv:517-opus4-408877

http://nbn-resolving.de/urn:nbn:de:kobv:517-opus4-408877 


\section{Kurzfassung}

Die vorliegende Arbeit beschäftigt sich mit Qualitätsmanagementsystemen in Nonprofit-Organisationen. Sie stellt dabei das Spannungsfeld verschiedener Akteursinteressen innerhalb von Nonprofit-Organisationen in den Vordergrund. Dies erfolgt anhand des mikropolitischen Ansatzes, der allen Akteuren innerhalb einer Organisation eigene Interessen zugesteht, die sie durch Taktiken und Strategien in Machtkämpfen versuchen durchzusetzen.

Untersucht wird der Prozess der Entstehung und Evaluation von konkreten Maßnahmen, den sogenannten Qualitätszielen, und den Einfluss von pädagogischen Mitarbeitenden auf deren Formulierung. Dies erfolgt anhand einer Einzelfallstudie. Mithilfe von qualitativen Interviews wurde untersucht, inwieweit pädagogische Mitarbeitende die Einflussmöglichkeiten des Qualitätsmanagementsystems zur strategischen Organisationsentwicklung und Durchsetzung eigener Interessen nutzen.

Die Ergebnisse zeigen, dass es zwei Typen von Mitarbeitenden gibt, aktive und passive, die entweder einen Machtgewinn oder -verlust erleben. Aufgrund der kooperativen Art der Kommunikation und Entscheidungsfindung sowie kaum divergierenden Interessen zwischen den verschiedenen Akteuren bleiben die vorhandenen Einflussmöglichkeiten im Sinne von organisationsinternen Machtkämpfen und mikropolitischen Taktiken bisher jedoch weitestgehend ungenutzt. Diese Falleigenschaften erklären, wieso der mikropolitische Ansatz bei der Analyse nicht zu den antizipierten Resultaten geführt hat.

\section{Abstract}

The topic of this paper is the role of quality management systems in non-profit organizations. The paper focuses on the potentially conflicting interests of different actors with within these organizations. The theoretical framework is based on the micro-political approach, drawing attention to actors' self-interest and how they apply tactics and strategies in intra-organizational power struggles.

Examined was the process of development and evaluation of concrete measures, so-called quality objectives, and the influence of pedagogical staff in their formulation. The research design was a single case-study. Qualitative interviews helped to understand to what extend the pedagogical staff used its influence opportunities to achieve own interests.

The results show two types of employees, active and passive, which gain or lose power. However, due to the cooperative communication and decision-making process as well as seldom divergent interests, intra-organizational power struggles do not take place and the actors do not need to use micro-political tactics. This case properties are the reason why the micro-political approach did not lead to the anticipated results. 


\section{Inhaltsverzeichnis}

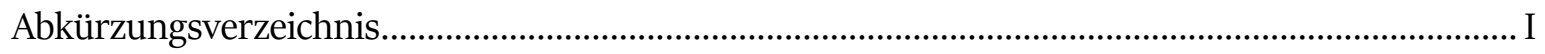

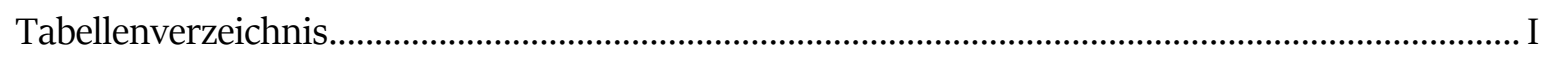

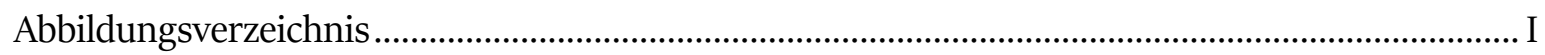

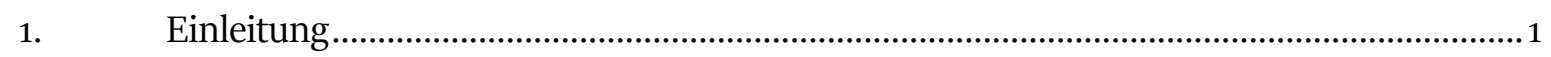

2. Qualitätsmanagement in der Freien Wohlfahrtspflege ................................................. 2

2.1. Modernisierungs- und Ökonomisierungsdruck in der Sozialwirtschaft .............................2

2.2. Konzepte der Qualitätssicherung und des Qualitätsmanagements für Nonprofit-

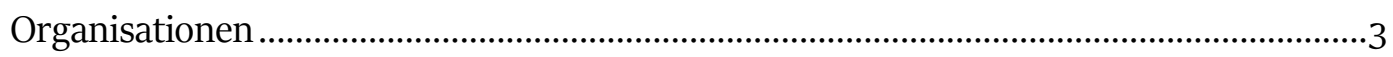

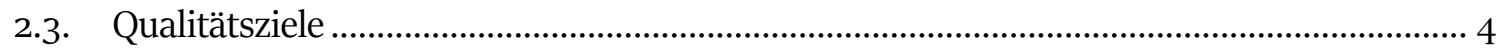

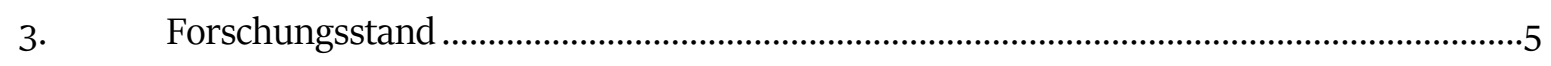

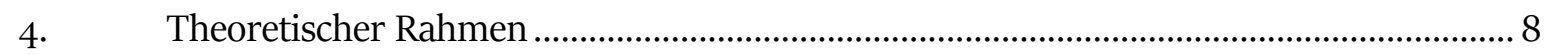

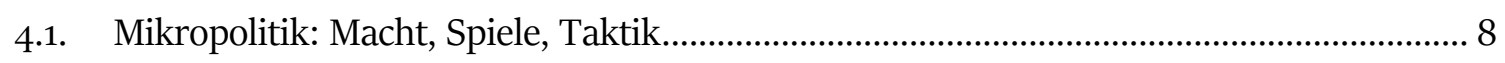

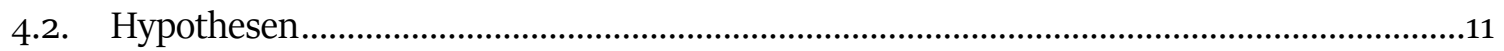

5. Forschungsdesign ...............................................................................................13

5.1. Fallbeispiel des Landesverbandes der Arbeiterwohlfahrt Berlin e.V. .................................13

5.2. Datenerhebung und Auswertung..................................................................................... 14

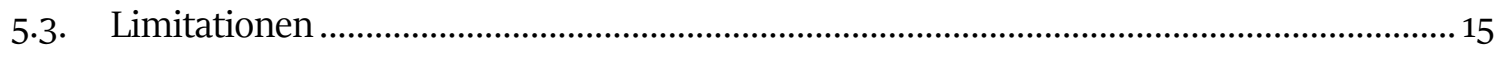

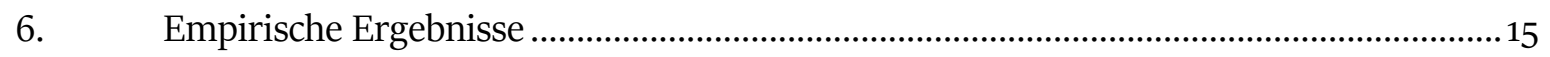

6.1. Einflussmöglichkeiten der pädagogischen Mitarbeitenden in organisationalen Ungewissheitszonen ....................................................................................................... 16

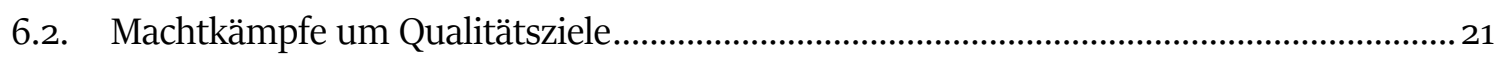

6.3. Anwendung mikropolitischer Taktiken............................................................................... 24

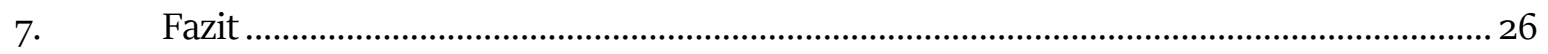

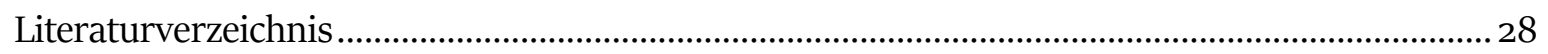




\section{Abkürzungsverzeichnis}

AWO Arbeiterwohlfahrt

BFD Bundesfreiwilligendienst

BSGH Bundessozialhilfegesetz

DIN Deutsches Institut für Normung e. V.

EFQM European Foundation for Quality Management

EQUASS European Quality in Social Services

FSJ Freiwilliges Soziales Jahr

NPO Nonprofit-Organisation

PQASSO Practical Quality Assurance System for Small Organisations

QB Qualitätsbeauftragte* $r$

QM Qualitätsmanagement

QMS Qualitätsmanagementsystem

SGB VIII Sozialgesetzbuch, Achtes Buch: Kinder- und Jugendhilfegesetz

SGB XI Sozialgesetzbuch, Elftes Buch: Soziale Pflegeversicherungsgesetz

\section{Tabellenverzeichnis}

Tabelle 1: Übersicht Interviewpartner*innen ............................................................................... 16

Tabelle 2: Häufigkeitstabelle Kategorie „QM-Perzeption“ und Unterkategorien............................. 17

Tabelle 3: Organisationale Ungewissheitszonen und Einflussmöglichkeiten - Gewiner*innen und Verlierer*innen durch und in QM............................................................................ 20

Tabelle 4: Ausformulierte Qualitätsziele am Beispiel „Nachhaltigkeit“ (top-down)....................... 22

Tabelle 5: Ausformulierte Qualitätsziele am Beispiel „Arbeitsklima“ (bottom-up) ......................... 23

\section{Abbildungsverzeichnis}

Abbildung 1: Formulierung von Qualitätszielen bei der AWO . 21

Abbildung 2: Formale Struktur und Entkopplung bei der Bearbeitung von Veränderungsmitteilungen in der Abteilung Freiwilligendienste 



\section{Einleitung}

In den vergangenen zwanzig Jahren hat die Soziale Arbeit in der Bundesrepublik Deutschland einen enormen Modernisierungs- und Ökonomisierungsdruck erlebt. Träger der Freien Wohlfahrtspflege müssen sich seit der Einführung neuer Finanzierungs- und Vertragsformen in einem verstärkten Wettbewerb mit anderen Nonprofit-Organisationen (NPOs) und privaten Anbietern behaupten (Grohs 2010, 415). Auf die veränderten Anforderungen an die Soziale Arbeit reagieren viele NPOs mit der Adaption von privatwirtschaftlichen Managementkonzepten wie beispielsweise dem Qualitätsmanagement (QM) (Bumbacher 1998, 342). Damit soll die Qualität der angebotenen sozialen Dienstleistungen sichergestellt und weiterentwickelt werden. Jährlich aufgestellte Qualitätsziele fungieren als Maßnahmen zur strategischen Qualitätsorientierung der NPOs (Bruhn 2013, 114).

Ein Großteil der Literatur zum Thema QM beschäftigt sich aus einer normativen Perspektive mit dem Aufbau von Qualitätsmanagementsystemen (QMS) in NPOs (Moldaschl 2001, 116; Bruhn 2013). In den vergangenen zehn Jahren sind jedoch auch einige empirische Studien zu qualitätsbezogenen Aktivitäten von NPOs erschienen (Vgl. Cairns u. a. 2005; Soltani, Lai, und Mahmoudi 2007; Liao, Soltani, und Yeow 2014; Melão und Guia 2015; Melão, Guia, und Amorim 2016). Die Implementierungsforschung beschäftigt sich mit den Einführungsgründen sowie Nutzen und Schwierigkeiten von verschiedenen Konzepten des QM für NPOs. Als kritischer Punkt wird die Involvierung der Mitarbeitenden gesehen: Sie erleben einen durch das QM herbeigeführten „Papierkrieg“ und reagieren auf die zusätzliche Arbeitsbelastung mit Unverständnis und mangelnder Überzeugung bis hin zu Ablehnung und offenem Widerstand gegenüber QM (Cairns u. a. 2005, 143; Melão und Guia 2015, 32of.; Melão, Guia, und Amorim 2016, 13f.). Der in der Empirie vorherrschende neoinstitutionalistische Ansatz ist zur Erklärung der in internen Akteuren begründeten Umsetzungsschwierigkeiten von QM nur begrenzt geeignet, da er das Verhältnis von Organisation und Umwelt betrachtet, aber innerorganisationale Prozesse außen vor lässt (Süß 2009).

Die vorliegende Arbeit stellt das Spannungsfeld verschiedener Akteursinteressen innerhalb von NPOs, in dem ein QMS bestehen muss, in den Vordergrund. Dies erfolgt mithilfe des mikropolitischen Ansatzes, der allen Akteuren innerhalb einer Organisation eigene Interessen zugesteht, die sie durch Taktiken und Strategien in Machtkämpfen versuchen durchzusetzen (Crozier und Friedberg 1979). Dieser Ansatz eignet sich als theoretischer Rahmen für diese Arbeit, da eine Organisation ihr Potential zur Qualitätsentwicklung erst dann voll ausschöpfen kann, wenn die Mitarbeitenden einen Mehrwert für die eigene sozialpädagogische Arbeit erleben und ihre Interessen befriedigt sehen. Aufgrund der Tatsache, dass Qualitätsziele als Soll-Zustand der eigenen Arbeit gelten (Arnold 2014, 597) und die Qualitätsgrundsätze der Organisation in konkreten Maßnahmen präzisieren, wird dem Prozess ihrer Entstehung und Evaluation eine besondere Bedeutung beigemessen. Die Forschungsfrage der vorliegenden Arbeit lautet daher: Welchen Einfluss haben pädagogische Mitarbeitende auf die Formulierung von Qualitätszielen? 
Zur Einordnung der Thematik gibt Kapitel 2 einen kurzen Überblick über die veränderten Rahmenbedingungen, in denen Soziale Arbeit heute stattfindet und die zur Einführung von Managementkonzepten in dieser Branche geführt haben. Anschließend werden grundlegende Konzepte der Qualitätssicherung und des QM für NPOs vorgestellt. Der aktuelle Forschungsstand zu Implementierung, Nutzen und Schwierigkeiten von QM in NPOs wird in Kapitel 3 diskutiert. Anschließend wird in Kapitel 4 der theoretische Rahmen der vorliegenden Arbeit dargestellt. Es werden zentrale Begriffe des mikropolitischen Ansatzes erläutert und aus der Theorie die im weiteren Verlauf zu untersuchenden Hypothesen aufgestellt. In Kapitel 5 wird das Forschungsdesign der Untersuchung vorgestellt, die Einzelfallstudie einer NPO am Beispiel des Landesverbandes der Arbeiterwohlfahrt Berlin. Kapitel 6 beinhaltet die Darstellung und Diskussion der empirischen Ergebnisse. Abschließend werden in Kapitel 7 Implikationen für Theorie und Praxis und weiterer Forschungsbedarf aufgezeigt.

\section{Qualitätsmanagement in der Freien Wohlfahrtspflege}

Zur besseren Einordnung der Forschungsfrage werden im folgenden Kapitel die Veränderungen dargestellt, welche in den vergangenen zwanzig Jahren zu einem enormen Modernisierungs- und Ökonomisierungsdruck in der Sozialen Arbeit führten. Anschließend werden zentrale Konzepte des QM vorgestellt, mithilfe derer NPOs versuchen die gegenwärtigen Herausforderungen zu meistern.

\subsection{Modernisierungs- und Ökonomisierungsdruck in der Sozialwirtschaft}

In den 199oer Jahren erfolgte eine weitgehende Neuordnung des Verhältnisses von Staat und freier Wohlfahrtspflege. Dies geschah einerseits durch eine veränderte Sozialgesetzgebung, andererseits durch die Übernahme von Logiken des New Public Management. Ziele waren dabei die Pluralisierung der Trägerstrukturen sowie eine verstärkte Wettbewerbsorientierung (Grohs 2010, 415).

Die gesetzlichen Neureglungen betreffen das Pflegeversicherungsgesetz (SGB XI), das Bundessozialhilfegesetz (BSGH) sowie das Kinder- und Jugendhilfegesetz (SGB VIII). Der Begriff der freien Träger umfasst nicht mehr ausschließlich Kirchen und Verbände der freien Wohlfahrtspflege ${ }^{1}$, sondern auch unabhängige Vereine, Initiativen und Projekte (§75 SGB VIII). Der Vorrang freier und privater Träger gegenüber öffentlichen Trägern konstituiert erstmalig die Gleichrangigkeit der beiden erst genannten (z. B. § 11 Abs. 2 SGB XI und §§ 10 und 93 BSHG) und hebt den bedingten Vorrang frei-gemeinnütziger NPOs auf (Butterwegge, Kutscha, und Berghahn 1999, 261).

\footnotetext{
${ }^{1}$ Hier sind die sechs Spitzenverbände der Freien Wohlfahrtspflege Arbeiterwohlfahrt (AWO), Deutscher Caritasverband (Caritas), Deutsches Rotes Kreuz (DRK), Diakonisches Werk der Evangelischen Kirche Deutschland (Diakonie), Paritätischer Wohlfahrtsverband und Zentralwohlfahrtsstelle der Juden gemeint, die in der Bundesarbeitsgemeinschaft der Freien Wohlfahrtspflege (BAGFW) zusammenarbeiten.
} 
Außerdem verlieren die Wohlfahrtsverbände ihren bisher vorherrschende prioritären Status bei der Erbringung sozialer Dienstleistungen (Grohs 2010, 414; Kulbach und Wohlfahrt 1996, 64). Diese sind nun der Konkurrenz weiterer, frei-gemeinnütziger und kommerzieller Anbieter ausgesetzt.

Zudem wurde die bisherige Zuwendungsfinanzierung nach dem Selbstkostendeckungsprinzip durch die Einführung von Leistungs- und Kostenvereinbarungen im BSHG und Teilen des SGB VIII abgelöst. Im Zuge des Neuen Steuerungsmodells wurden auf kommunaler Ebene Instrumente des Kontraktmanagements und Qualitätsentwicklungsvereinbarungen eingeführt (Grohs 2010, 415). Die Erbringung sozialer Dienste soll nun in Anlehnung an einen durch Preis und Qualität gesteuerten Wettbewerb stattfinden (Butterwegge, Kutscha, und Berghahn 1999, 262). Diese Entwicklungen können als „Ökonomisierung“ des Sozialsektors und der Bildung von „Quasi-Märkten“ zusammengefasst werden (Butterwegge, Kutscha, und Berghahn 1999, 265f.).

\subsection{Konzepte der Qualitätssicherung und des Qualitätsmanagements für Nonprofit- Organisationen}

Die veränderten Rahmenbedingung bei der Erbringung sozialer Dienstleistungen - neue Finanzierungs- und Vertragsformen sowie verstärkter Wettbewerb auch mit privaten Anbietern - hatten einen Anstieg von internen und externen Qualitätsanforderungen an NPOs zur Folge (Bumbacher 1998, 343). Einerseits erfordert die neue Wettbewerbssituation eine Fokussierung der NPOs auf die Qualität ihrer angebotenen Dienstleitungen zur Sicherung bzw. Verbesserung der Marktposition (Butterwegge, Kutscha, und Berghahn 1999, 266). Andererseits erhöhen die Notwendigkeit des Nachweises von Qualität selbst oder explizit geforderte Maßnahmen zur Sicherstellung und Entwicklung von Qualität (§§ 78 a-g SGB VIII) den Legitimationsdruck (Beckmann u. a. 2004, 17).

Jedoch existiert bisher kein einheitlicher, allgemein akzeptierter Qualitätsbegriff als Maßstab (Arnold 2014, 588; Melão und Guia 2015, 314). Zurückgegriffen werden kann auf die weit verbreitete Definitionen des Deutschen Instituts für Normung e. V. (DIN): Hier bezeichnet Qualität „den Grad, in dem ein Satz inhärenter Merkmale [des Produktes/der Dienstleistung] Forderungen erfüllt" (DIN EN ISO 9000:2005). Im Kontext sozialer Dienstleistungen ist Qualität keine objektive Größe (Kulbach und Wohlfahrt 1996, 103), sondern ergibt sich aus den Merkmalen der realisierten Beschaffenheit der Dienstleitung und aus der Wahrnehmung der Leistung, d. h. dem Erfüllungsgrad von Anforderungen/Erwartungen der Leistungsempfangenden (Bruhn 2013, 19f.). Da NPOs dienstleistungsähnliche Leistungen erbringen, können für sie Qualitätsansätze für Dienstleistungsorganisationen angewendet werden (Bumbacher 1998, 344f.). Im Rahmen der Qualitätssicherung müssen Qualitätsstandards als anerkannte Kriterien oder Normen des beruflichen Handelns entwickelt und durch geeignete Maßnahmen umgesetzt und überprüft werden (Kulbach und Wohlfahrt 1996, 104). Orientierung bieten die Kategorien Strukturqualität, Prozessqualität und Ergebnisqualität (Kulbach und Wohlfahrt 1996, 105; Merchel 2004, 144; Arnold 2014, 590). Die Strukturqualität beinhaltet die Rahmenbedingungen und den effektiven und 
angemessenen Mitteleinsatz von den zur Verfügung stehenden finanziellen, materiellen und personellen Ressourcen (Beckmann u. a. 2004, 19) für die Mitarbeitenden und Kunden (interne Struktur) sowie bei der Vernetzung mit Behörden, Institutionen und anderen Organisationen (externe Struktur). Die Prozessqualität bezieht sich auf alle Aktivitäten des Leistungserbringungsprozesses, d. h. alle Arbeits- und Entscheidungsabläufe zwischen Mitarbeitenden und Kunden. Die Ergebnisqualität fragt nach der Zielerreichung: Sie zeigt sich z. B. in der durch die Leistungserbringung bewirkten Veränderungen und kann anhand von Soll-Ist-Vergleichen ermittelt werden (Wirkungskontrolle) (Kulbach und Wohlfahrt 1996, 105ff.).

Um den Forderungen nach Beschreibung, Sicherstellung, Bewertung und Weiterentwicklung von Qualität nachzukommen, haben zahlreiche NPOs QMS eingeführt. Unter einem QMS versteht man die Gesamtheit der qualitätsbezogenen Zielsetzungen und Tätigkeiten innerhalb einer Organisation, u. a. die Planung, Lenkung, Prüfung und Dokumentation von Qualität (Arnold 2014, 593). Weit verbreitet ist die Zertifizierung nach den DIN EN ISO gooo ff. Qualitätsnormen. Auf Grundlage der Normenreihe, welche Mindestanforderungen an ein QMS festlegt und für sämtliche Organisationen unabhängig von deren Größe oder Branche anwendbar ist, wird ein organisationsspezifisches QMS aufgebaut. Den Qualitätsnormen liegt ein prozessorientierter Ansatz des QM zugrunde: Prozesse werden anhand ihrer Ist-Werte mit den Soll-Werten verglichen und entdeckten Abweichungen werden im Rahmen des Plan-Do-Check-Act-Zyklus durch Verbesserungen und Veränderungen entgegengewirkt (Bruhn 2013, 158ff.). Weiterhin gibt es ein von der European Foundation for Quality Management entwickeltes ganzheitliches Unternehmensmodell im Sinne des Total-Quality-Management (EFQM-Modell) (Arnold 2014, 596). Zudem existieren branchenspezifische Konzepte, z. B. auf europäischer Ebene die zweistufige Zertifizierung European Quality in Social Services (EQUASS) (Melão, Guia, und Amorim 2016, 5f.) oder in Großbritannien das Practical Quality Assurance System for Small Organisations (PQASSO) (Cairns u. a. 2005, 138). Diese Modelle haben gemeinsam, dass bei der Anwendung von QM in der Sozialen Arbeit der Schwerpunkt auf der Struktur- und Prozessqualität und weniger auf der Zielerreichung liegt (Arnold 2014, 590).

\subsection{Qualitätsziele}

Zur Weiterentwicklung von Qualitätsstandards und der langfristigen, strategischen Qualitätsorientierung von NPOs ist die Bestimmung von Qualitätszielen unerlässlich (Bruhn 2013, 97). Innerhalb der Qualitätsplanung sind Qualitätsziele - nach der Ermittlung der Übereinstimmung von angebotener und wahrgenommener Leistungsqualität (Ist-Zustand) - der entwickelte Soll-Zustand, welchen die NPO mithilfe einer geeigneten Strategie erreichen will (Arnold 2014, 597).

Qualitätsziele lassen sich unterscheiden in marktgerichtete Ziele (z. B. Kundenbindung und Steigerung der Kundenzufriedenheit), organisationsgerichtete Ziele (z. B. Qualitätsbewusstsein der haupt-/ehrenamtlichen Mitarbeitenden, Effizienzsteigerung der Prozesse) und gesellschaftsgerichtete Ziele (z. B. Verteilungsgerechtigkeit und langfristige Sicherung der Leistungserstellung). Ziele müssen in ihrem Inhalt, Ausmaß und Zeitbezug so konkret und ggf. messbar formuliert 
sein, dass anschließend die Zielerfüllung überprüft werden kann (Bruhn 2013, 117). Erschwerend für eine generalisierte Zielformulierung und -überprüfung im Sinne von Ergebnisqualität ist die aus den zwei wesentliche Merkmale der Sozialen Arbeit - individuelle und alltagsorientierte Ausrichtung (sozial)pädagogischen Handelns - resultierende Zieldiffusität sozialer Dienstleistungen (Merchel 2004, 139). Merchel nennt als Probleme u. a. den „Mangel an eindeutigen Ursache-Wirkung-Beziehungen, Individualität der Ziele (...), koproduktiven Charakter der Leistungserstellung“ (2004, 145).

Bei der Bestimmung und Entwicklung von Qualität und Qualitätszielen müssen demnach die Perspektiven von Management, Mitarbeitenden und Adressaten berücksichtigt werden. Deren Vorstellungen von Qualität unterscheiden sich abhängig von der organisationalen Positionierung der jeweiligen Akteure sowie ihrer fachlichen und/oder persönlichen Hintergründe und folgen verschiedenen Präferenzen und Rationalitäten (Beckmann u. a. 2004, 18). Bruhn ordnet die Festlegung von lang- und kurzfristigen Qualitätszielen als Aufgabe des Vorstandes ein (2013, 114). Zugleich empfiehlt er die Vorschläge und Ideen aller Mitarbeitenden und Ehrenamtlichen einzubeziehen, z. B. dadurch, dass die Aufgabe der Erarbeitung von Qualitätsstandards und -zielen an Qualitätszirkel delegiert wird (Bruhn 2013, 117).

\section{Forschungsstand}

Die QM-Literatur hat zumeist normativen Charakter: Es gibt zahlreiche Handbücher mit Beschreibungen idealtypischer QMS und Erläuterungen von best practice Beispielen (Moldaschl 2001, 116; Vgl. Bruhn 2013; Bumbacher 1998). Empirische Studien zur Implementierung und Anwendung von QM sind im Vergleich zu anderen Managementkonzepten im Dritten Sektor bislang weniger weit verbreitet. Die Autoren weisen auf eine geringe Aufmerksamkeit der Qualitätsforschung gegenüber NPOs (Cairns u. a. 2005, 137), dem Freiwilligensektor (Liao, Soltani, und Yeow 2014, 1296) bzw. der Sozialwirtschaft (Melão und Guia 2015, 314) hin. Ergebnisse von Umfragen und vergleichenden Fallstudien zu qualitätsbezogenen Aktivitäten in der Privatwirtschaft und im öffentlichem Sektor liegen bereits seit den 1990er Jahren vor (Powell 1995; Westphal, Gulati, und Shortell 1997; Waldman u. a. 1998; Kennedy und Fiss 2009). Der Nonprofit-Sektor ist jedoch erst in den vergangenen zehn Jahren Gegenstand mehrerer Untersuchungen mit qualitativem oder mixed-methods Forschungsdesigns geworden (Cairns u. a. 2005; Soltani, Lai, und Mahmoudi 2007; Liao, Soltani, und Yeow 2014; Melão und Guia 2015; Melão, Guia, und Amorim 2016). Die Implementierungsforschung befasst sich schwerpunktmäßig mit den Gründen für die Einführung von QMS, positiven und negativen Auswirkungen der Implementierung und der Rolle von QM als Erfolgsfaktor für die Organisation. Die Ergebnisse unterscheiden sich dabei je nach untersuchtem QM-Modell nur gering.

Als Einführungsgründe werden expliziter und antizipierter Druck seitens staatlicher Geldgeber, die Notwendigkeit, die eigene Glaubwürdigkeit und Legitimität zu erhöhen sowie das Bedürfnis, Verantwortung gegenüber den zahlreichen internen und externen Stakeholdern zu zeigen, 
identifiziert (Cairns u. a. 2005, 140). Die NPOs wollen dem gestiegenen externen Druck mit Qualitätsverbesserungen ihrer Dienstleitungen Stand halten und Exzellenz zeigen (Melão, Guia, und Amorim 2016, 8). Zwar besteht die Dualität aus dem Streben nach ökonomischen und sozialen Gewinnen durch die Einführung des QMS weiterhin, jedoch tritt die Maxime des Effizienzstrebens gegenüber dem Bedürfnis nach Legitimität in den Hintergrund (Kennedy und Fiss 2009, 907f.). Finanzielle Unterstützung, wie z. B. durch den Europäischen Sozialfond, kann den letzten Anstoß zur Einführung von branchenspezifischen QMS wie EQUASS geben (Melão, Guia, und Amorim 2016, 9). Bei den bekannteren Konzepten wie DIN EN ISO 9001 spielt das Image und Prestige, welches durch die Zertifizierung gewonnen wird, eine große Rolle (Melão und Guia 2015, 319).

Bei der Implementierung selbst werden Auswirkungen auf die Struktur- und Prozessqualität wahrgenommen. Die Ergebnisqualität hingegen wird, wie im ersten Kapitel bereits erläutert, kaum oder nur indirekt beeinflusst. Als internen Nutzen nennen englische NPOs „organisationale Selbstreflexion, Lernen und Entwicklung“ (Cairns u. a. 2005, 141). Mit der im Rahmen der DIN EN ISO 9001-Einführung stattgefundenen (Neu-)Strukturierung von Prozessen und Verantwortlichkeiten konnte eine verbesserte interne Kommunikation und eine gesteigerte Motivation der Mitarbeitenden erreicht werden (Melão und Guia 2015, 319). Diese Aussagen sind konsistent mit denen der Untersuchung von EQUASS in portugiesischen NPOs (Melão, Guia, und Amorim 2016). Dieses Modell führt ferner zu einer Verbesserung der internen Arbeitsabläufe im Vergleich zum EFQM und zu einer höheren Kundenorientierung im Vergleich zu DIN EN ISO 9001. Aus Sicht der NPOs übertreffen die internen Nutzen die externen deutlich (Melão, Guia, und Amorim 2016, 9). Die von Melão, Guia und Amorim befragten NPOs nehmen als externen Nutzen eine höhere Servicequalität und Kundenzufriedenheit wahr (2016, 11f.). Dies steht im Kontrast zu den Ergebnissen von Cairns, Harris, Hutchison und Tricker, die keinen direkten oder messbaren Nutzen für die Kunden finden konnten $(2005,148)$. Diese Wirkung könnte gegebenenfalls langfristig als Resultat von einer verstärkte Kommunikation und Beziehung zu den Kunden auftreten (Melão und Guia 2015, 320). Als großen Vorteil sehen die NPOs ihr gesteigertes Image und ihre Reputation gegenüber der organisationalen Umwelt (Cairns u. a. 2005, 141; Melão und Guia 2015, 319). Die angestrebten Verbesserungen von Performanz, Wirtschaftlichkeit und Ergebniswirkung der Dienstleistungen bleiben hingegen aus (Melão, Guia, und Amorim 2016, 1307f.; Melão, Guia, und Amorim 2016, 16).

Eine wesentliche Schwierigkeit stellt der mangelnde Einsatz der Mitarbeitenden aufgrund von Unverständnis der QM-Maßnahmen und die mangelnde Überzeugung ihrer Sinnhaftigkeit dar (Cairns u. a. 2005, 143). Die sogenannte QM-Bürokratie (Standardisierung der Prozesse, Erstellung von Handbücher, Nutzung von Formblättern) führt zu einer erhöhten Arbeitsbelastung sowohl für das Management als auch für die pädagogischen Mitarbeitenden. Ein Mangel an Zeit und finanziellen Ressourcen bestärkt weiterhin die Ablehnung bis hin zu Widerstand gegenüber den durch QM bedingten Veränderungen (Cairns u. a. 2005, 143; Melão und Guia 2015, 320f.; Melão, Guia, und Amorim 2016, 13f.). Hohe externe Audit-Kosten und eine neu zu erlernende Fachsprache sind insbesondere bei kleinen NPOs ein weiteres Hindernis (Melão und Guia 2015, 321). 
Interessanterweise berichten NPOs, welche häufiger externe Beweggründe zur Einführung von QMS nannten, zugleich weniger Vorteile und mehr Schwierigkeiten der Implementierung (Melão, Guia, und Amorim 2016, 16).

Um QM als Erfolgsfaktor zu etablieren, müssen Strategien zur Überwindung dieser zumeist in Personen begründeten Umsetzungsschwierigkeiten wie einer „widerwilligen, feierlichen Ehrerbietung“ (Cairns u. a. 2005, 148) bis hin zu offenem Boykott, entwickelt werden. Eine Kultur der offenen Organisation und insbesondere das Empowerment der Mitarbeitenden als immaterieller Faktoren sind notwendig für ein gelingendes QM (Powell 1995, 28). Insofern ist es verwunderlich, weshalb Studien, welche die Qualitätserwartungen verschiedener Stakeholder an NPOs analysieren, die hauptamtlichen Mitarbeitenden außen vor lassen (Jones und Shandiz 2015) oder diesen eine sehr geringe Aufmerksamkeit entgegenbringen. Liao, Soltani und Yeow fordern, gezielt den Blickwinkel der Mitarbeitenden näher zu beleuchten um Barrieren zu überwinden (2014, 1311).

Es stellt sich weiterhin die Frage, ob Enttäuschung und Unzufriedenheit über die Ergebnisse von QM auf konzeptionelle Schwachstellen oder Implementierungsdefizite zurückzuführen sind (Soltani, Lai, und Mahmoudi 2007, 165). Moldaschl bemängelt, dass die zahlreichen normativen Darstellungen modellimmanente Unzulänglichkeiten ausblenden und Probleme nur in der Anwendung ausmachen $(2001,116)$.

Die Forschung betrachtet die Institutionalisierung von QM oder anderen Managementkonzepten meist aus der neoinstitutionalistischen Perspektive (Vgl. Tolbert und Zucker 1983; Westphal, Gulati, und Shortell 1997; Cairns u. a. 2005; Kennedy und Fiss 2009; Liao, Soltani, und Yeow 2014). Der Neoinstitutionalismus hat das Verhältnis von Organisation und Umwelt zum Gegenstand. Die Organisation orientiert ihr Handeln nicht an den Kriterien Effizienz und Effektivität, sondern an den Erwartungen der Umwelt, um sich Legitimität und ein angemessenes Image zu verschaffen (Vgl. Walgenbach und Meyer 2008). Die Unzulänglichkeiten dieses Theorieansatzes liegen in der mangelnden Betrachtung von organisationsinternen Prozessen (Süß 2009). Diese Prozesse unterscheiden sich in NPOs mit ihrer „charakteristischen Kultur, diffusen Machtstrukturen, unterschiedlichen Werten, zahlreichen Stakeholdern und vielfachen Zielen“ (Cairns u. a. 2005, 148) von den Prozessen in der Privatwirtschaft, in welcher das Konzept QM entwickelt wurde.

Insofern ist es unumgänglich sich auf die Suche nach neuen Erklärungsansätzen zu begeben, anstatt die negativen Effekte von QM lediglich mit einer schlechten Umsetzung des Konzeptes zu begründen. Es ist ein Theorieansatz erforderlich, der sich mit den Prozessen innerhalb von Organisationen beschäftigt und die Mitarbeitenden als eigenständige Akteure begreift. Im nachfolgenden Kapitel werden daher die Grundzüge des mikropolitischen Ansatzes der Organisationsforschung dargelegt. Basierend auf diesem theoretischen Ansatz werden anschließend anhand eines Fallbeispiels die Ursachen für die geschilderten Widersprüchlichkeiten beleuchtet und die Rolle der Mitarbeitenden sowie ihren Einfluss auf das QMS näher betrachtet. 


\section{Theoretischer Rahmen}

Innerhalb der Auswertung der Literatur konnte gezeigt werden, dass es bisher wenig überzeugende Erklärungen für die Abweichung des „gelebten“ vom geplanten QM in NPOs gibt. Dieses Kapitel beschäftigt sich daher mit den Aussagen des mikropolitischen Ansatzes zur Erklärung von Prozessen innerhalb der NPOs während der Implementierung und nachfolgenden Institutionalisierung von QMS. Der Fokus soll dabei auf dem Einfluss der pädagogischen Mitarbeitenden auf die Formulierung von Qualitätszielen innerhalb der Organisationen liegen.

\subsection{Mikropolitik: Macht, Spiele, Taktik}

Mikropolitische Ansätze zur Erklärung von Verhalten in Organisationen finden sich in der Politikwissenschaft (Bogumil und Schmid 2001), der Betriebswirtschaftlehre (Moldaschl 2001), aber insbesondere in der Organisationssoziologie (Crozier und Friedberg 1979) und Organisationspsychologie (Neuberger 1995; Blickle 2004; Neuberger 2006). Unter Mikropolitik versteht man Spiele bzw. Kämpfe um (Macht-)Ressourcen in der organisatorischen Arena, bei denen Akteure ihre Interessen durch Strategien und Taktiken verfolgen. Die Besonderheit ist dabei, dass diese Kämpfe unterhalb der „großen“ Politik bzw. den formellen Beziehungen innerhalb der Organisation stattfinden (Moldaschl 2001, 115).

Der mikropolitische Ansatz ist akteurszentriert. Es wird auf die Annahme verzichtet, dass innerhalb einer Organisation nur eine einzige Strategie existiert, mithilfe derer die Umsetzung eines bestimmten Konzeptes oder die Durchführung eines Reorganisations-/Innovationsprozesses gegen partikulare Widerstände durchgesetzt werden muss (Moldaschl 2001, 116). Stattdessen wird angenommen, dass eine Vielzahl von Akteuren und Interessen existiert (Crozier und Friedberg 1979, 57). Zur Durchsetzung ihrer Interessen benötigen die Akteure Macht. Crozier und Friedberg definieren Macht als die „verfügbare Möglichkeit, auf andere Individuen oder Gruppen einzuwirken“ $(1979,39)$ und ein bestimmtes Verhalten im Sinne der eigenen Interessen zu erzeugen. Darauf aufbauend ist Einfluss die Realisierung von Macht. Ziel ist eine „erfolgreiche Beeinflussung anderer zum Realisieren von Zielen und Plänen einer Person im Handlungskontext einer Organisation“ (Blickle 2004, 82f.). Hierfür müssen die Akteure zueinander in Beziehung stehen, innerhalb derer sie versuchen ein gemeinsames Ziel zu erreichen. Aufgrund dessen sind die beteiligten Akteure voneinander abhängig und zwischen ihnen entwickelt sich eine implizite oder explizite Austausch- bzw. Verhandlungsbeziehung (Crozier und Friedberg 1979, 39).

Der Handlungskontext einer Organisation ist gekennzeichnet von ihren strukturellen Merkmalen, d. h. den Bedingungen und Zwängen unter denen die Verhandlungen stattfinden. Bereiche, in denen weniger Strukturen und Regeln herrschen, bezeichnen Crozier und Friedberg als „organisationale Ungewissheitszonen“ (1979, 47). Die Akteuren versuchen die vorhandenen Handlungsspielräume zu nutzen und zu kontrollieren - d.h. Macht auszuüben - um ihre Interesse durchzusetzen (Crozier und Friedberg 1979, 47). Crozier und Friedberg beschreiben die folgenden vier organisationalen Ungewissheitszonen als Quellen der Macht: Expertenwissen, Umweltbeziehungen, Informationskontrolle und Hierarchie (1979, 51ff.; vgl. Bogumil und Schmid 2001). 
Expertenwissen bezieht sich auf den für das Funktionieren der Organisation erforderlichen Sachverstand, dessen Aneignung für die übrigen Mitarbeitenden Kosten verursachen würde. Umweltbeziehungen bezeichnen Kenntnisse und Fähigkeiten zur Kontrolle von Kontakten zu internen und externen Akteuren oder zwischen Organisation und Umwelt in Form von Vermittlung oder Übersetzung unterschiedlicher Handlungslogiken. Die Kontrolle von Informations- und Kommunikationskanälen, z. B. zwischen organisationalen Einheiten, lässt sich mit dem Prinzip des gatekeepers verdeutlichen. Hierarchie und organisationale Regeln sollen das Verhalten der Akteure vorhersehbar machen und die drei zuvor genannt organisationalen Ungewissheitszonen minimieren. Zugleich bieten sie neue Verhandlungsspielräume für untergebene Mitarbeitende und Vorgesetzte, da eine Organisation für gewöhnlich nicht durch ,Dienst nach Vorschrift' arbeitsfähig ist. Der mikropolitische Ansatz kann Erklärungen zur Entstehung, Stabilisierung und Veränderung von Machtbeziehungen liefern.

Konkret entfalten sich diese Machtbeziehungen im Rahmen von Spielen oder Machtkämpfen, deren Ablauf offen und unvorhersehbar, aber dennoch nicht spontan ist (Crozier und Friedberg 1979, 56). Die Akteure verfolgen teilweise eine rationale Strategie um mithilfe ihrer vorhandenen Macht einen höchstmöglichen „Gewinn“ innerhalb der Organisation zu erzielen. Ihr Verhalten kann offensiv oder defensiv sein: Sie können zur Durchsetzung der eigenen Forderungen selbst Zwang ausüben oder durch den systematischen Schutz des eigenen Spielraumes versuchen, sich dem Zwang der anderen Akteure zu entziehen (Crozier und Friedberg 1979, 56). Der Erfolg ihrer Strategie ist abhängig von ihren Gegenspielern, deren Strategien und Machtmitteln (Moldaschl 2001, 117). Es gibt zwei Kategorien von Spielen, Routine- und Innovationsspiele, die jeweils unterschiedliche „Regeln der Sinnkonstitution und der Legimitation (...) zur Mobilisierung von Ressourcen“ haben (Bogumil und Schmid 2001, 67). Routinespiele beziehen sich auf den regulären Organisationsalltag und sind gekennzeichnet von „Beständigkeit, Zuverlässigkeit und inkrementellen Verbesserungen“, während Innovationsspiele die Spielregeln und Gewinne verändern und die Akteure zwingen neue Strategien zu entwickeln (Bogumil und Schmid 2001, 68).

Von den oben beschriebenen Interessen- und Machtdisparitäten ausgehend, können Organisationen als politische Arenen verstanden werden, in denen die Akteure ihre Interessen in Verhandlungen durchzusetzen versuchen und miteinander Kompromisse schließen (Göbel 1999, 8f.). Die konvergierenden oder divergierenden Interessen der Akteure führen dabei zur Entstehung von Konfliktlinien oder der Bildung von Koalitionen (Moldaschl 2001, 117). In der organisatorischen Arena nutzen die Akteure mikropolitische Taktiken. Damit gemeint sind Handlungsmuster und -sequenzen, welche „die Zielperson zum Werkzeug der oft unausgesprochenen, zuweilen getarnten Interessen des Akteurs machen wollen“ (Neuberger 2006, 85). Blickle hat anhand empirischer Untersuchungen der organisationspsychologischen Forschung 13 repräsentative, mikropolitische Taktiken zusammengefasst, welche Arbeitnehmende und Führungskräfte nutzen können um ihren Einfluss geltend zu machen (2004, 83): 
- Assertivität:

Anweisungen geben, Forderungen stellen, Fristen setzen, auf Vorschriften pochen, die Befolgung gebieten;

- Blockieren:

Die Einstellung der Zusammenarbeit androhen, die andere Person ignorieren, die Freundschaft beenden, langsamer arbeiten;

- Sanktionen/Belohnung:

Eine Lohnerhöhung zurückhalten/versprechen, mit Entlassung drohen, mit einer schlechten Leistungsbeurteilung drohen/eine gute Leistungsbeurteilung versprechen;

- Tauschangebot:

Einen kleinen Gefallen oder große Unterstützung anbieten, an einen geleisteten Gefallen erinnern;

- Einschmeicheln:

Fremdaufwertung: Komplimente machen, loben, freundlich sein; Selbstaufwertung: sich verbal als beliebt, umgänglich oder verträglich darstellen; Zustimmung zeigen, kleine Gefälligkeiten erweisen;

- Rationalität:

Logische Argumente vortragen, detaillierte schriftliche Ausarbeitungen vorlegen, unterstützende Informationen geben;

- Koalitionsbildung:

Die Unterstützung der Kolleg*innen oder Mitarbeitenden aktivieren;

- Höhere Instanzen einschalten:

Sich an höhere Vorgesetzte wenden;

- Inspirierende Appelle:

Appelle an Emotionen, Werte oder Ideale, um Begeisterung hervorzurufen;

- Konsultation:

Den Einflussadressaten nach seiner Meinung fragen, um Vorschläge bitten;

- Legitimation:

Auf die eigene Autorität oder die Regeln der Organisation pochen;

- Persönliche Appelle:

An die Gefühle der Freundschaft und Loyalität appellieren;

- Self-Promotion:

Sich als kompetent, tüchtig und/oder erfolgreich präsentieren.

Mehrere Taktiken zusammen ergeben eine Strategie. Darunter versteht Neuberger ein langfristiges, „leitendes Prinzip“ (policy) des Akteurs zur „Selektion, Kombination oder Sequenz von Taktiken“, deren vorausgeplantes Aufeinanderfolgen „eine Ordnung oder ein Muster“ bildet (2006, 107). 


\subsection{Hypothesen}

Der mikropolitische Ansatz kann im Folgenden relevante Erklärungen zu den in der Literatur dargestellten erheblichen internen Umsetzungsschwierigkeiten von QM in NPOs liefern. Es ergibt sich zunächst die Frage, in welcher Form sich die Haltung der pädagogischen Mitarbeitenden auf das QM in NPOs auswirkt, wenn sie das Konzept QM nicht verstehen, von dessen Sinnhaftigkeit wenig überzeugt sind oder keine Zeit haben sich damit auseinanderzusetzen (Cairns u. a. 2005, 143; Melão und Guia 2015, 32of.; Melão, Guia, und Amorim 2016, 13f.). Weiterhin ist zu klären, welchen Einfluss die Mitarbeitenden auf das „gelebte“ QMS haben. Welchen Einfluss haben die Mitarbeitenden auf die Formulierung von Qualitätszielen, die als Ausdruck des Selbstverständnisses der NPO und der Auseinandersetzung mit den internen und externen Anforderungen an den Inhalt der eigenen Arbeit verstanden werden? Schlussendlich, welche Strategien und Taktiken nutzen pädagogische Mitarbeitende um ihre Interessen bei der Formulierung von Qualitätszielen innerhalb der NPO geltend zu machen?

Als interessierende Subjekte werden nachfolgend alle hauptamtlichen Mitarbeitenden als Akteure im Sinne des mikropolitischen Ansatzes verstanden. In NPOs arbeiten Menschen mit unterschiedlichen (Ausbildungs-)Hintergründen, z. B. aus der Sozialarbeit, Pädagogik, Psychologie, Volksund Betriebswirtschaftslehre. Je nach Funktion, Aufgaben- und Tätigkeitsspektrum vertreten diese Akteure verschiedenste Interessen. Sie vertreten zwar auch Eigeninteressen, überwiegend arbeiten sie aber im Sinne verschiedener Anspruchsgruppen, z. B. ihrer Kund*innen bzw. Klient*innen und deren Angehörigen, der Organisations-/Vereinsmitglieder, der Geldgeber oder der Gesellschaft. Die Vielzahl individueller Rationalitäten sind nur teilweise mit dem vermeintlich übergeordneten Organisationszweck kongruent (Kühl 2001, 79f.). Erschwerend kommt die generelle Zielpluralität bei NPOs im Bereich der sozialen Dienstleistungen hinzu (Merchel 2004, 139). Dadurch können sich je nach konkreter Fragestellung unterschiedliche Akteurskonstellationen und Koalitionen innerhalb der NPO ergeben.

Die dem QM zugrunde liegenden Prinzipien, z. B. Formalisierung und Standardisierung von Arbeitsabläufen, führen zu einer teils explizit intendierten Veränderung der Machtverhältnisse (Moldaschl 2001, 116). Prozessfestschreibungen und Prozesstransparenz verringern den Handlungsspielraum und die persönliche Entscheidungsfreiheit der pädagogischen Mitarbeitenden, da von ihnen die Einhaltung von Verfahrens- und Arbeitsabläufen gefordert wird. Während bisher das „Wohl der Klient*innen“ (anstelle der Kund*innen) und die eigene Fachlichkeit als Qualitätskriterium im Vordergrund standen, ist inzwischen verstärkt Effizienz der Maßstab (Dahme u. a. 2008, 188). Das drückt sich in einer „an oberflächlichen Kosten-Nutzen-Kalkülen ausgerichtete[n] ökonomische[n], im Kern technokratische[n] Rationalität“ aus (Merchel 2004, 140). Es gibt weniger organisationale Ungewissheitszonen, da insgesamt mehr Strukturen und Regeln innerhalb der NPO herrschen, und weniger Möglichkeiten, Machtressourcen wie Expertenwissen, Umweltbeziehungen, Informationskontrolle oder formale Regeln einzusetzen, und Macht zu realisieren. Es ergibt sich die folgende, erste Fallhypothese: 
H1a: Die Einführung von QMS verringert organisationale Ungewissheitszonen und wirkt sich negativ auf die Einflussmöglichkeiten der Mitarbeitenden aus.

Zugleich entsteht bei der Neuverhandlung von Qualität „eine an kommunikativen Prozessen der Verständigung und an einer kooperativen Problembewältigung ausgerichteten Rationalität“ mit dem Ziel den individuellen und organisationalen Ansprüchen auf Fachlichkeit und Professionalität gerecht zu werden (Merchel 2004, 140). QM stellt sich als Innovationsspiel mit veränderten Spielregeln dar (Bogumil und Schmid 2001, 68) durch welches organisationale Ungewissheitszonen vergrößert werden. Für die Mitarbeitenden bietet diese Situation durch den Einsatz von Machtressourcen Chancen auf neue Gewinne bzw. eine veränderte Gewinnaufteilung. Alternativ formuliere ich die folgende Fallhypothese:

H1b: $\quad$ Die Einführung von QMS vergrößert bzw. erzeugt neue organisationale Ungewissheitszonen und wirkt sich positiv auf die Einflussmöglichkeiten der Mitarbeitenden aus.

Unabhängig davon, ob sich ihre Einflussmöglichkeiten vergrößern oder verringern, versuchen die pädagogischen Mitarbeitenden in den organisatorischen Machtkämpfen ihre verfügbaren Machtressourcen einzusetzen und „Trumpfkarten“ (Erfahrungswissen, verdeckte Spielräume und Leistungsreserven) auszuspielen, um ihre Interessen durchzusetzen (Kühl 2001, 93). Hohes Potential für Machtkämpfe innerhalb der NPO birgt die Formulierung von Qualitätszielen, welche Teil der strategischen Planung der NPO ist (Bruhn 2013, 97). Es ist unklar, mit welcher Intensität diese Machtkämpfe ablaufen. Hierbei kommt es auf die sich durchgesetzte Rationalität innerhalb des QMS und den Prozess der Zielformulierung an. Es ergibt sich eine weitere Fallhypothese:

H2: $\quad$ Wenn die Einführung von QMS die Machtverhältnisse innerhalb der NPO verändert, entwickelt sich die Formulierung von Qualitätszielen zu einem Machtkampf zwischen den beteiligten Akteuren und ihren divergierenden Interessen.

Qualitätsziele zu erarbeiten ist oftmals Aufgabe der Qualitätszirkel (Bruhn 2013, 112). Neben den formellen Verbesserungsmechanismen bietet die Beteiligung an entscheidender Stelle innerhalb des QMS den pädagogischen Mitarbeitenden die Möglichkeit, ihre Interessen mittels geeigneter mikropolitischer Taktiken und Strategien geltend zu machen. Welche der zuvor beschriebenen Taktiken sie konkret nutzen (können), ist unklar. Dafür wird die folgende Fallhypothese formuliert:

H3: $\quad$ Wenn sich die Formulierung von Qualitätszielen als Machtkampf darstellt, dann werden alle Akteure mikropolitische Taktiken nutzen, um ihre Interessen durchzusetzen.

Im empirischen Teil dieser Arbeit werden diese Fragestellungen anhand eines Fallbeispiels überprüft. Im folgenden Kapitel wird zunächst das Forschungsdesign vorgestellt. 


\section{Forschungsdesign}

Dieses Kapitel hat die Erläuterung der methodischen Vorgehensweise zum Gegenstand. Dies beinhaltet die Begründung der Fallauswahl, der genutzten Methoden zur Datengewinnung und Datenauswertung sowie das Aufzeigen möglicher Limitationen des Forschungsdesigns.

Zur Untersuchung des Phänomens ,Akteursbezogene Schwierigkeiten der QM-Umsetzung in NPOs' bietet sich ein qualitatives Vorgehen an, um dem Anspruch des „Verstehen[s] von Handlungsmotiven“ gerecht zu werden (Hopf 2016, 161). Entgegen der vorherrschenden Meinung, Aufgabe der qualitativen Sozialforschung sei es Hypothesen zu generieren und sie habe sich demzufolge am unbedingten Prinzip der theoretischen Offenheit zu orientieren (Brüsemeister 2008, 48), wurde sich hier in Anlehnung an Hopf für ein theoriegeleitetes und hypothesentestendes Vorgehen entschieden (2016). Insbesondere bei der Prüfung von Hypothesen über singuläre Tatbestände wie die „Eigenheiten der Struktur und Funktionsweise einzelner Organisationen“ sind offene, qualitative Verfahren nützlich (Hopf 2016, 158). Vor dem Hintergrund von Theorie und Literatur kann die Erhebung und Auswertung qualitativer Daten „mit mehr Genauigkeit, Umsicht und Selbstreflexion als vielfach üblich“ erfolgen (Hopf 2016, 164).

Als Untersuchungsform wurde die Einzelfallstudie ausgewählt. Ziel und Vorteil von Fallstudien ist, das interessierte Phänomen in einer realen Umwelt zu untersuchen. Die genaue Beschreibung des Falles ermöglicht eine Identifikation von „relevanten Einflussfaktoren“ und eine „Interpretation von Zusammenhängen“ (Mayring 2008, 42). Ziel ist es, die „Deutungs- und Handlungsmuster der einzelnen Mitglieder [der Organisation] miteinander zu vergleichen“ (Brüsemeister 2008, 56). Die Auswahl des Untersuchungsgegenstandes erfolgte mithilfe eines theoretischen Samplings (Brüsemeister 2008, 21). Das Fallbeispiel sollte eine NPO sein, welche soziale Dienstleistungen erbringt, professionell arbeitet, hauptamtliche Mitarbeitende mit unterschiedlichen (Ausbildungs-)Hintergründen vereint und bereits seit einigen Jahren Erfahrung mit der Anwendung eines QMS hat.

\subsection{Fallbeispiel des Landesverbandes der Arbeiterwohlfahrt Berlin e.V.}

Als Fallbeispiel ausgewählt wurde der Landesverband der Arbeiterwohlfahrt (AWO) Berlin e.V. Die AWO ist ein unabhängiger Spitzenverband der Freien Wohlfahrtspflege in Deutschland. Der Landesverband der AWO Berlin ist einer von 30 Landes- und Bezirksverbänden. Ferner gibt es sieben Berliner Kreisverbände, die Tochtergesellschaft AWO pro:mensch gGmbH und ca. 8o korporative Mitgliederorganisationen ${ }^{2}$.

Durch die Magdeburger Beschlüsse der Bundeskonferenz 2007³ sind AWO-Einrichtungen verpflichtet ein integriertes QMS einzuführen. Das AWO Tandemkonzept beinhaltet eine

\footnotetext{
${ }^{2}$ Siehe https://www.awoberlin.de/AWO-auf-einen-Blick-8.43033.html (abgerufen am 15.08.2016)

${ }^{3}$ Siehe http://www.awo-informationsservice.org/uploads/media/AWO_Magdeburger_Beschluesse_2007.pdf (abgerufen am 18.08.2016)
} 
Zertifizierung nach DIN EN ISO 9001:2008 und den AWO Normen. In Berlin wurde der Kreisverband Südost als „Vorreiter“ im Jahr $2008^{4}$ erfolgreich zertifiziert. Es folgten weitere Kreisverbände und im Dezember 2012 auch der Landesverband. Im AWO Landesverband arbeiten insgesamt ca. 140 Mitarbeitende: 40 in der Zentrale (Geschäftsführung, Lohn-, Finanzbuchhaltung, einige Referent*innen) und die restlichen Mitarbeitenden in Abteilungen an dezentralen Standorten in Berlin.

Die Analyseeinheit der vorliegenden Arbeit ist die Abteilung Freiwilligendienste anhand derer exemplarisch der Einfluss der pädagogischen Mitarbeitenden auf die Formulierung der Qualitätsziele untersucht werden soll. Aufgabe der Abteilung ist es, Jugendliche und jungen Erwachsene, die ein Freiwilliges Soziales Jahr (FSJ) oder einen Bundesfreiwilligendienst (BFD) in einer Einrichtung der AWO oder der korporativen Mitgliederorganisationen absolvieren, zu betreuen. Dazu zählt neben der Vermittlung von FSJ- und BFD-Plätzen die Regelung aller vertraglichen und finanziellen Angelegenheiten und die pädagogische Begleitung des Freiwilligendienstes in Form von Reflexionsgesprächen und 25 Bildungstagen ${ }^{5}$. In der Abteilung Freiwilligendienste arbeiten zehn Mitarbeitende in der pädagogischen Koordination, vier in der Verwaltung sowie zwei im Management.

\subsection{Datenerhebung und Auswertung}

Als Datenerhebungstechnik wurden Leitfadengespräche genutzt (Schnell, Hill, und Esser 2005, 378). Es erfolgten teilstrukturierte, 20-45-minütige Gespräche mit drei pädagogischen Mitarbeitenden, dem dezentralen QM-Beauftragten der Abteilung Freiwilligendienste (QB) und dem Leiter des QM im Landesverband (siehe Tabelle 1). Im Interview sollten die Befragten „möglichst frei zu Wort kommen, um einem offenen Gespräch nahe zu kommen“ (Mayring 2008, 67). Mithilfe eines Interview-Leitfadens wurde sichergestellt, dass alle forschungsrelevanten Punkte angesprochen wurden (Schnell, Hill, und Esser 2005, 387). Die pädagogischen Mitarbeitenden sollten sich zu folgenden Themen äußern: Ihren bisherigen Erfahrungen mit und ihrer Beurteilung von QM, den aktuellen Qualitätsziele, deren Erarbeitung, Monitoring und Evaluation, ihren Möglichkeiten eigene Ideen und Vorstellungen einzubringen sowie was sie anders machen würde, wenn QM heute noch einmal neu eingeführt werden würde. Der Qualitätsbeauftragte der Abteilung Freiwilligendienste und der Leiter des QM des Landesverbandes sollten sich zusätzlich dazu äußern, welchen Eindruck sie von der Beurteilung des QM durch die Mitarbeitenden haben und wie sie das Funktionieren des QM innerhalb der Abteilung Freiwilligendienste im Vergleich zu anderen Abteilungen beurteilen. Die Interviewaussagen konnten anhand der Einsichtnahme in QM-Dokumente (Formblätter, Verfahrensabläufe und Arbeitsanweisungen, Qualitätsziele) und den Eindrücken einer eigenen Teilnahme an einem Qualitätszirkel und einem internen Audit ergänzt werden. Die

\footnotetext{
${ }^{4}$ Siehe http://www.awo-südost.de/qualitaetsmanagement (abgerufen am 15.08.2016)

${ }^{5}$ https://www.awoberlin.de/FSJ-Freiwilliges-Soziales-Jahr-843084.html (abgerufen am 15.08.2016)
} 
Interviews wurden mitgeschnitten (2,5 Stunden) und anschließend vollständig transkribiert (30 Seiten Text) (Schnell, Hill, und Esser 2005, 388).

Die Auswertung der Daten erfolgte in Anlehnung an die qualitative, strukturierende Inhaltsanalyse nach Mayring (2010, 92ff.). Aus der Theorie wurden deduktiv die ersten Kategorien abgeleitet und anschließend bei der Sichtung des Materials weitere Kategorien induktiv entwickelt und hinzugefügt (Mayring 2010, 83). Die Datenauswertung erfolgte mithilfe der Analysesoftware f4analyse.

\subsection{Limitationen}

Der Gültigkeitsbereich der nachfolgend vorgestellten Ergebnisse erstreckt sich nur auf das hier untersuchte Fallbeispiel, es besteht „keine statistische Repräsentativität [und eine] schwierige Übertragbarkeit der Befunde“ auf andere Organisationen (Atteslander 2008, 56). Generell sind beim Vorgehen einer qualitativen Einzelfallstudie die zentralen Gütekriterien empirischer Sozialforschung Reliabilität (Zuverlässigkeit) und Validität (Gültigkeit) ein kritischer Punkt (Mayring 2010, 116f.). Anstatt einer Generalisierbarkeit ist eine Typisierung der Daten im Sinne der „Identifizierung (...) fallinterner Merkmale“ möglich (Brüsemeister 2008, 29f.).

Es kann von einem Auswahleffekt ausgegangen werden, da vermutlich mehr Mitarbeitende mit ausgeprägt negativen oder positiven Emotionen gegenüber QM bereit waren ein Interview zu führen. Weiterhin können Antwortverzerrungen auftreten, z. B. (vorgetäuschte) Meinungslosigkeit, Zustimmungstendenzen oder die Abgabe sozial erwünschter Antworten „als situationsspezifische Reaktion auf die Datenerhebung“ (Schnell, Hill, und Esser 2005, 332), da sich die Interviewten der Brisanz des Themas QM für die Organisation bewusst sind.

Bei der Auswertung wurde durch ein mehrfaches Codieren der Interviewdaten und dem Vergleich der Kodierungen die Stabilität der Ergebnisse erhöht (Intracoderreliabilität) (Mayring 2010, 117). Um eine intersubjektive Nachvollziehbarkeit und Reproduzierbarkeit der Analyse zu ermöglichen, sind die vollständigen Transkripte der Interviews, das erarbeitete Kategoriensystem sowie der Ergebnisexport der Datenauswertung auf der CD beigefügt (Brüsemeister 2008, 132).

\section{Empirische Ergebnisse}

Die Abteilung Freiwilligendienste kann auf eine vierjährige Erfahrung mit dem integrierten QMS nach DIN EN ISO und AWO Normen zurückblicken. Im Team herrscht ein grundlegendes Verständnis für die Notwendigkeit von QM und eine Akzeptanz oder zumindest eine Duldung der mit QM einhergegangenen Standardisierung von Prozessen. Der Leiter des QM des Landesverbandes Herr Q. bescheinigt den Freiwilligendiensten im Vergleich zu anderen Abteilungen einen sehr hohen Implementierungs- und Nutzungsgrad:

„Geben wir ihnen eine acht [auf einer Skala von eins bis zehn]. Das hat etwas mit dem Setting zu tun und nachhaltig mit der Zusammenarbeit der Leitung und dem QB dort vor Ort.“ 
Tabelle 1: Übersicht Interviewpartner*innen

\begin{tabular}{|c|c|c|c|c|c|}
\hline $\begin{array}{l}\text { Interview- } \\
\text { partner*in }\end{array}$ & Frau A. & Herr B. & Herr $\mathbf{C}$. & Herr D. & Herr Q. \\
\hline Abteilung & \multicolumn{4}{|c|}{ Freiwilligendienste } & $\begin{array}{l}\text { Landes- } \\
\text { verband }\end{array}$ \\
\hline Funktion & \multicolumn{3}{|c|}{ Pädagogische Koordination } & $\begin{array}{c}\text { Sachbearbeiter } \\
\text { und QB }\end{array}$ & Leiter QM \\
\hline Ausbildung & $\begin{array}{c}\text { Bachelor } \\
\text { Soziale } \\
\text { Arbeit }\end{array}$ & $\begin{array}{c}\text { Magister } \\
\text { Politik- } \\
\text { wissenschaft }\end{array}$ & $\begin{array}{l}\text { Berufs- } \\
\text { ausbildung } \\
\text { Erzieher }\end{array}$ & $\begin{array}{l}\text { Diplom- } \\
\text { Ingenieur }\end{array}$ & $\begin{array}{c}\text { Diplom- } \\
\text { Sozialarbeiter }\end{array}$ \\
\hline $\begin{array}{l}\text { Arbeits-be- } \\
\text { ginn }\end{array}$ & 2015 & 2015 & 2011 & 2011 & 2008 \\
\hline QM-Zirkel & Nein & $\mathrm{Ja}$ & Ja & Ja, als QB & Ja, als QB \\
\hline
\end{tabular}

Quelle: Eigene Erhebung und Darstellung.

\subsection{Einflussmöglichkeiten der pädagogischen Mitarbeitenden in organisationalen Ungewissheitszonen}

Die erste Fallhypothese bezieht sich auf die Veränderung von organisationalen Ungewissheitszonen durch die QM-Einführung und einem daraus resultierenden Machtverlust oder -gewinn für die Mitarbeitenden. In Kapitel 4.1 wurden vier organisationale Ungewissheitszonen als Machtquellen beschrieben: vorhandenes Expertenwissen, die Kontrolle von Umweltbeziehungen, die Kontrolle von Informations- und Kommunikationskanälen sowie formale, organisationale Regeln und Hierarchie.

Unter dem Aspekt Expertenwissen wurde nicht nur das Verständnis des Konzeptes und der Funktionsweise von QM durch die Mitarbeitenden betrachtet, sondern auch den Grad ihrer Involvierung in und ihrer Begeisterung für QM. Die pädagogischen Mitarbeitende, mit denen die Interviews geführt wurden, lassen sich unterscheiden in Herr B. und Herr C. als begeisterte und engagierte Mitarbeitende, die aktiv im QMS involviert sind und freiwillig am Qualitätszirkel der Abteilung Freiwilligendienste teilnehmen, und Frau A. als teilweise aufgeschlossene, aber meist gleichgültig bis offen ablehnende Mitarbeitenden, die sich passiv zeigt und ihre Mitarbeit im QMS auf das Nötigste beschränkt. Die aktiven Mitarbeitenden empfinden QM größtenteils als hilfreich, vereinfachend und sinnvoll, die passive Mitarbeitende als anstrengend, zeitraubend und überfordernd (siehe Tabelle 2). Die Unterscheidung der Mitarbeitenden anhand ihrer QM-Perzeption beschreibt der Leiter des QM folgendermaßen:

„Man hat ungefähr 20\% Begeisterte, (...) das sind die Strukturierten. Die freuen sich wenn sie früh hinkommen, die bekommen einen Maßnahmenkatalog, das ist cool, da steht alles drin, was ich machen muss. (...) 6o\%, die sind eher Mitläufer. Die machen halt das, was gemacht werden 
muss. Die finden das so in Ordnung, aber braucht man auch nicht unbedingt, würde auch anders gehen. (...) Und dann hast du die $20 \%$, die das wirklich ganz schlimm finden. Die das als Einengung ihrer Persönlichkeit finden, ihres gesamten kreativen Prozesses. Die sagen: 'Die ganzen Verrückten da oben, was lassen die sich da schon wieder einfallen?!'.“

Tabelle 2: Häufigkeitstabelle Kategorie „QM-Perzeption“ und Unterkategorien

\begin{tabular}{|l|c|c|c|}
\hline QM-Perzeption & Frau A. & Herr B. & Herr C. \\
\hline anstrengend/zeitraubend & 7 & 0 & 0 \\
\hline hilfreich/einfach & 0 & 4 & 4 \\
\hline sinnvoll & 1 & 0 & 5 \\
\hline Überforderung & 6 & 0 & 0 \\
\hline effizient/effektiv & 2 & 0 & 2 \\
\hline QM-Typ & passiv & & aktiv \\
\hline
\end{tabular}

Quelle: Eigene Erhebung und Darstellung.

Durch die Einführung von QM verringert sich zunächst die organisationale Ungewissheitszone Expertenwissen für alle Mitarbeitenden. Mitarbeitende, die bereit und fähig dazu sind, das System zu verstehen und in den gegebenen Strukturen mitzuarbeiten, haben im anschließenden Spiel QM einen entscheidenden Vorteil: Ihr angeeignetes Expertenwissen ist die Grundlage auf der Informationskontrolle und Nutzung von Hierarchien zur Durchsetzung ihrer Interessen möglich ist. Den übrigen Mitarbeitenden mangelt es an den Kenntnissen der Spielregeln oder sie haben kein Interesse am Spiel teilzunehmen und haben folglich von vornherein keine Chance zu gewinnen.

Für die Analyse der Umweltbeziehungen wurde betrachtet, bei welchen Tätigkeiten externe Akteure involviert sind. Das Selbstverständnis der Interviewten dafür ist interessant: Als intern wird nur die Abteilung Freiwilligendienste verstanden, als extern bereits die räumlich getrennte Zentrale (von den Interviewten als AWO Landesverband bezeichnet) sowie die Freiwilligen, deren Einsatzstellen und andere involvierte Institutionen. Einen Machtverlust erleben die Mitarbeitenden bei sämtlichen Bürotätigkeiten. Die QM-Dokumente (Formblätter, Verfahrensabläufe, Arbeitsanweisungen) werden entweder direkt vom Landesverband vorgegeben oder müssen nach Erarbeitung durch die Abteilung vom QM des Landesverbandes und der Geschäftsführung freigegeben werden. Die durch die QM-Dokumente minimierten organisationalen Ungewissheitszonen werden allerdings als Erleichterung und als für die Arbeitsorganisation im Büro hilfreich und sinnvoll angesehen. Die Vereinheitlichung führt zur Vereinfachung von Arbeitsabläufen insbesondere bei der Einarbeitung neuer Mitarbeitender oder dem Wiedereinstieg nach längerer Abwesenheit (Kategorie QM-Perzeption, Unterkategorien hilfreich/einfach, sinnvoll). Der Machtverlust wird auch von der im QMS passiven Mitarbeitenden Frau A. als etwas Positives wahrgenommen: „Deshalb glaube ich schon, wenn standardisiert ist, das auf jeden Fall sehr, sehr sinnvoll ist." 
Notwendig ist der Machtverlust auch aus einem ganz pragmatischen Grund: dem Selbsterhaltungstrieb der Freiwilligendienste. Ein zertifiziertes QMS ist Bedingung für die Förderung seitens der Senatsverwaltung, die einen Großteil der Finanzierung von FSJ und BFD ausmacht:

„Die sagen, ohne QM-System läuft hier nichts mehr. Werden Zuwendungen nicht mehr ausgezahlt oder wie auch immer. Oder es gibt Schwierigkeiten“ (QB Herr D.)

Vom QM-Standards unberührt ist die Kernaufgabe der pädagogischen Koordination, die Begleitung der Freiwilligen während der Seminare. Herr B. begründet mit dem „koproduktiven Charakter der Leistungserstellung“ (Merchel 2004, 145):

„Ein ganz wichtiger Grund ist, dass wir bei der Seminargestaltung ganz stark auf die Partizipation der Teilnehmenden eingehen wollen und das kann man schlecht mit einem QMStandard, was an Inhalten vorkommen soll.“

QM vergrößert zwar die organisationalen Ungewissheitszonen in diesem Fall nicht, führt aber im Vergleich zu den Bürotätigkeiten zu keiner Verringerung durch zusätzliche Strukturen und Regeln für die pädagogische Arbeit. Die Mitarbeitenden erleben im Kontakt zu ihren Freiwilligen keinen Machtverlust, sondern es wird weiterhin ihrer Fachlichkeit vertraut.

Bei der Analyse der Informationskontrolle ist auf die relevanten Informations- und Kommunikationskanäle von QM einzugehen. Von zentraler Bedeutung ist die Kommunikation innerhalb des Qualitätszirkels. Wer in diesem involviert und engagiert ist, hat einen entscheidenden Informationsvorteil und bessere Möglichkeiten eigene Interessen zu platzieren. Die Mitglieder des Qualitätszirkels erfahren zuerst von den QM-Neuerungen der Geschäftsführung und des QM des Landesverbandes und beraten darüber, wie sinnvoll es ist, Dokumente des Landesverbandes in das Register des Freiwilligendienstes zu übernehmen. Außerdem entscheiden sie, ob Veränderungsmitteilungen aus der Abteilung stattgegeben wird. Da der Qualitätszirkel ebenfalls eine tragende Rolle in der Formulierung der Qualitätsziele einnimmt (siehe Fallhypothese 2), haben dessen Mitglieder einen entscheidenden Vorteil gegenüber den übrigen Mitarbeitenden ihre Interessen potenziell durchzusetzen.

Zuletzt spielen Hierarchie und formale, organisationale Regeln als Verhandlungsgegenstand zwischen Mitarbeitenden und Vorgesetzten vor dem Hintergrund der grundlegenden Regeländerungen innerhalb der Organisation durch das QMS eine zentrale Bedeutung. Der Grundgedanke des regelgeleiteten QM ist den Frei- und Handlungsspielraum der Mitarbeitenden zu begrenzen und die Macht der Leitung zu erhöhen. Allerdings schafft jede organisationale Regel neue Ungewissheit darüber, wie die Akteure in der Praxis mit dieser umgehen (Vgl. Bogumil und Schmid 2001, 58). Die Einflussmöglichkeiten der Mitarbeitenden lassen sich nach der Art ihres Agierens unterscheiden. Einzelne Mitarbeitende können mithilfe des Instruments der Veränderungsmitteilung dem QMS neue Impulse geben, da die Qualitätsbeauftragten „verpflichtet [sind] jeder Meldung zu einer Veränderung nachzugehen“ (Herr Q.). Größere Macht haben die Mitarbeitenden im Kollektiv, z. B. im Qualitätszirkel oder als Abteilung. Indem sie die Einhaltung formaler, organisationaler Regeln fordern und die in der bottom-up Logik erarbeiteten Qualitätsziele im Sinne einer 
strategischen, mittelfristigen Ausrichtung der Abteilung nutzen, können die Mitarbeitenden ihre Interessen gegenüber der Geschäftsführung geltend machen. Für die Geschäftsführung wird es schwer sein, die durch das Expertenwissen der Abteilung artikulierten Interessen zur Sicherstellung und Weiterentwicklung von Qualität auszuschlagen. Diese Ziele können markt-, organisations- oder gesellschaftsgerichtet sein, z. B. die Erhöhung des Mietzuschusses für die Freiwilligen, eine Senkung der Betreuungsquote oder die Öffnung des Freiwilligendienstes für geflüchtete, junge Erwachsene. Der Leiter des QM Herr Q. bemängelt:

„Die [Mitarbeitenden] haben viel mehr Macht. Die könnten viel, viel mehr positive Kraft entfalten. Themensetting, Beschwerden, Entwicklung.“

Dies ist aber erst im fortgeschrittenen Stadium der Implementierung möglich, denn die Mitarbeitenden benötigen Wissen und Ressourcen als kritische Faktoren, um ihre Einflussmöglichkeiten auszuschöpfen. Insbesondere die untere Ebene wird durch QM aber erst einmal gelähmt. Die Mitarbeitenden erleben einen enorm begrenzen Handlungsspielraum und Belastung durch die QMDokumente (Kategorie QM-Perzeption, Unterkategorie anstrengend/zeitraubend, Überforderung) und eine erhöhte Kontrolle durch die Fokussierung der internen und externen Audits auf ihrer Ebene. Die einzelnen Mitarbeitenden sind folglich derartig damit beschäftigt, den formellen Anforderungen von QM an die Organisation und Dokumentation ihrer Arbeit gerecht zu werden, dass sie gar keine Zeit haben als Kollektiv ihre Interessendurchsetzung zu organisieren.

Zusammenfassend lassen sich für alle organisationalen Machtquellen Gewinner*innen und Verlierer*innen feststellen (siehe Tabelle 3). Im ersten Schritt erleben alle Mitarbeitenden einen Machtverlust durch QM. Ihre organisationalen Ungewissheitszonen werden verringert, da sie sich den für das Funktionieren von QM nötigen Sachverstand erst einmal aneignen müssen, um das Spiel QM überhaupt mitspielen zu können. Die Mitarbeitenden werden durch die Vorgaben der Senatsverwaltung und des Landesverbandes zur prozessgerechten Organisation und Dokumentation der Bürotätigkeiten, die als „notwendiges Übel“ zu ihrer pädagogischen Arbeit dazugehören, verpflichtet und erleben eine weitere Begrenzung ihres Handlungsspielraumes. Fallhypothese H1a („Die Einführung von QMS verringert organisationale Ungewissheitszonen und wirkt sich negativ auf die Einflussmöglichkeiten der Mitarbeitenden aus.") wird somit im Allgemeinen für alle Mitarbeitenden bestätigt. In einem zweiten Schritt jedoch erleben die Mitarbeitenden, welche immer noch Freude am QM-Spiel empfinden, einen Machtgewinn durch QM. Sie können sich freiwillig im Qualitätszirkel einbringen und dort über Veränderungsmitteilungen entscheiden, Qualitätsziele formulieren und Agendasetting betreiben. Mitarbeitende, die bis zur Endrunde mitspielen, haben dann wesentlich mehr Möglichkeiten ihre Interessen durchzusetzen. Fallhypothese H1b („Die Einführung von QMS vergrößert bzw. erzeugt neue organisationale Ungewissheitszonen und wirkt sich positiv auf die Einflussmöglichkeiten der Mitarbeitenden aus.") muss somit im Allgemeinen abgelehnt werden, kann aber im Speziellen nur für die aktiven Mitarbeitenden bestätigt werden. 
Tabelle 3: Organisationale Ungewissheitszonen und Einflussmöglichkeiten - Gewiner*innen und Verlierer*innen durch und in QM

\begin{tabular}{|c|c|c|}
\hline $\begin{array}{l}\text { Macht- } \\
\text { quelle }\end{array}$ & $\begin{array}{l}\text { "Gewinner*innen“" } \\
\text { (erleben Machtgewinn) }\end{array}$ & $\begin{array}{l}\text { „Verlierer*innen“ } \\
\text { (erleben Machtverlust) }\end{array}$ \\
\hline $\begin{array}{l}\text { Experten- } \\
\text { wissen }\end{array}$ & $\begin{array}{l}\text { Im OMS aktive Mitarbeitende: } \\
\text { - } \quad \text { Haben QM verstanden } \\
\text { - } \quad \text { Sind an QM interessiert, sitzen frei- } \\
\text { willig im Qualitätszirkel } \\
\text { - } \quad \text { Empfinden QM größtenteils als hilf- } \\
\text { reich, vereinfachend und sinnvoll }\end{array}$ & $\begin{array}{l}\text { Im QMS passive Mitarbeitende: } \\
\text { - Verstehen QM nicht } \\
\text { - } \text { Sind nicht interessiert an QM } \\
\text { - Empfinden QM größtenteils } \\
\text { als anstrengend, zeitraubend } \\
\text { und überfordernd }\end{array}$ \\
\hline $\begin{array}{l}\text { Umwelt- } \\
\text { beziehun- } \\
\text { gen }\end{array}$ & $\begin{array}{l}\text { Pädagogische Seminararbeit: } \\
\text { - Partizipation der Teilnehmenden als } \\
\text { Qualitätskriterium legitimiert Ab- } \\
\text { wesenheit von QM-Standards für } \\
\text { diesen Bereich }\end{array}$ & $\begin{array}{l}\text { Büroarbeit: } \\
\text { - Vorgabe von QM-Dokumente } \\
\text { aus Landesverband } \\
\text { - Notwendigkeit von QMS, da } \\
\text { Zuwendungen der Senatsver- } \\
\text { waltung sonst nicht gezahlt } \\
\text { werden }\end{array}$ \\
\hline $\begin{array}{l}\text { Informa- } \\
\text { tions- } \\
\text { kontrolle }\end{array}$ & 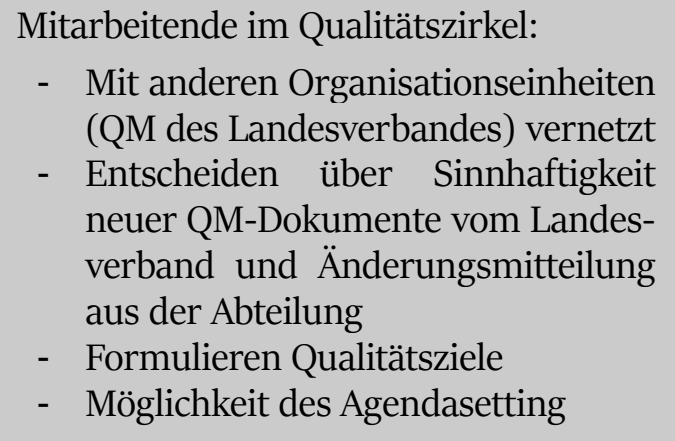 & $\begin{array}{l}\text { Mitarbeitende nicht im Qualitäts- } \\
\text { zirkel: } \\
\text { - Keine direkte Kommunikation } \\
\text { mit anderen Organisations- } \\
\text { einheiten }\end{array}$ \\
\hline $\begin{array}{l}\text { Hierar- } \\
\text { chie }\end{array}$ & $\begin{array}{l}\text { Einzelne Mitarbeitende: } \\
\text { - Können nächsthöhere Instanzen } \\
\text { zwingen sich mit Veränderungsmit- } \\
\text { teilung auseinanderzusetzen } \\
\text { Mitarbeitende im Kollektiv (Qualitätszir- } \\
\text { kel insgesamt): } \\
\text { - Höhere Durchsetzungskraft von ge- } \\
\text { meinsamen Interessen in Form von } \\
\text { Qualitätszielen } \\
\text { - Entwicklung strategische Ausrich- } \\
\text { tung der Abteilung unter Qualitäts- } \\
\text { gesichtspunkten }\end{array}$ & $\begin{array}{l}\text { Mitarbeitende in unterer Ebene: } \\
\text { - } \text { Begrenzung Handlungsspiel- } \\
\text { raum (bis zur Lähmung der } \\
\text { operativen Arbeit) durch QM- } \\
\text { Dokumente } \\
\text { - } \begin{array}{l}\text { Kontrolle durch interne und } \\
\text { externe QM-Audits }\end{array}\end{array}$ \\
\hline $\begin{array}{l}\text { Insge- } \\
\text { samt }\end{array}$ & $\begin{array}{l}\text { Mitarbeitende, die aktiv im QMS und } \\
\text { Mitglied des Qualitätszirkels sind } \rightarrow \\
\text { Können ihre Interessen leichter artiku- } \\
\text { lieren und eher durchsetzen }\end{array}$ & $\begin{array}{l}\text { Mitarbeitende, die passiv im QMS } \\
\text { und kein Mitglied des Qualitätszir- } \\
\text { kels sind } \rightarrow \text { Können ihre Interes- } \\
\text { sen nicht/nur schwer artikulieren } \\
\text { und kaum durchsetzen }\end{array}$ \\
\hline
\end{tabular}

Quelle: Eigene Erhebung und Darstellung. 


\subsection{Machtkämpfe um Qualitätsziele}

Qualitätsziele innerhalb der AWO werden anhand von zwei Logiken formuliert: Durch vorgegebene Leitthemen zur Qualitätsentwicklung des ganzen Verbandes (top-down) und durch die Orientierung an den Bedürfnissen der Einheiten (bottom-up; siehe Abbildung 1). Die top-down Logik beinhaltet Themen des Bundesverbandes, die über ein Rundschreiben an die Bundesgeschäftsführer*innenkonferenz vorgegeben und bei der Entwicklung von konkreten Qualitätszielen berücksichtigt werden sollen. Innerhalb der bottom-up Logik besteht die Forderung, dass sich die Mitarbeitenden in den Einheiten mit der Qualitätsentwicklung vor Ort auseinandersetzen und geeignete Qualitätsziele mit konkreten Maßnahmen und Messgrößen zur Bewertung der Umsetzung formulieren sollen.

Abbildung 1: Formulierung von Qualitätszielen bei der AWO

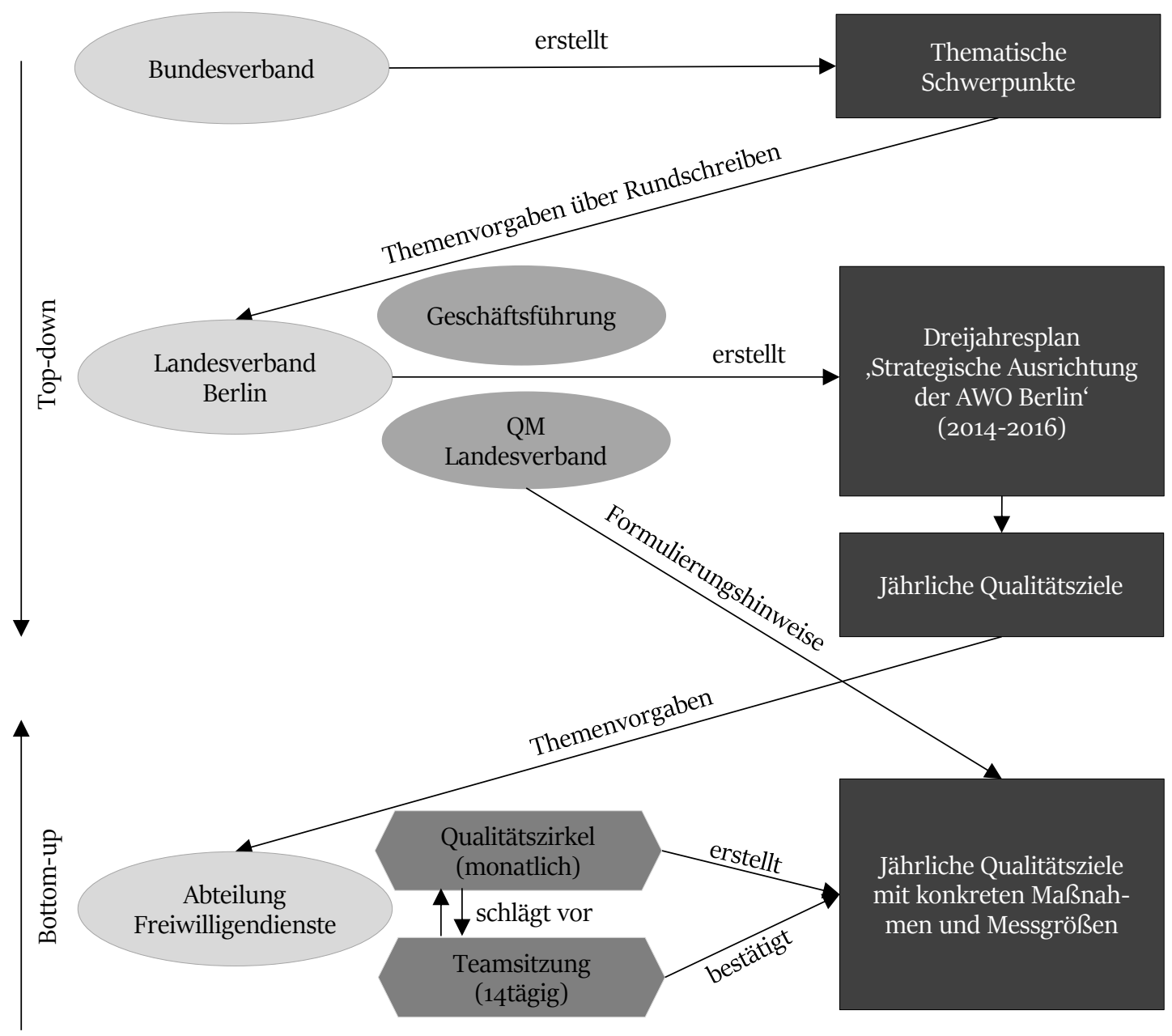

Quelle: Eigene Erhebung (Interviewaussagen) und Darstellung.

Es gibt verschiedene Spielrunden: zunächst innerhalb der Abteilung in der Teamsitzung, dann innerhalb des Qualitätszirkels und anschließend in Zusammenarbeit mit dem QM des Landesverbandes. Herr B. nennt als Ausgangspunkt von Qualitätszielen „Ideen und Anregungen, die auf Teamsitzungen gemeinsam überlegt werden“. Anschließend fließen die von der Basis hervorgebrachten Themen ebenso wie die Vorschläge des Landesverbandes in die Zielformulierung ein. 
„[Danach] stellen [wir] diese wiederum in der Teamsitzung vor. Da können sie noch einmal verändert werden“, erklärt Herr C. die Zusammenarbeit mit dem Team. Außerdem betont er den kooperativen Charakter des Spiels ,Qualitätsziele entwickeln': „Wir sprechen miteinander anstatt übereinander, das wird im Team sehr basisdemokratisch gelebt. "Zuletzt folgt eine Rückkopplung mit dem QM des Landesverbandes, welche Hinweise zur Formulierung der Qualitätsziele und ihrer Mess- und Prüfbarkeit gibt. Herr Q. erklärt:

„Wir machen keine Vorgaben, wie und in welcher Art die Ziele in den Einrichtungen umgesetzt werden. Aber wir machen Vorgaben zu den Themen, zu den Bezügen. Da wollen wir, dass die Kollegen kreativ sind und die jeweiligen Ziele anpassen auf die Bedingungen vor Ort.“

Der angenommene Machtkampf um Qualitätsziele scheint sich bei der AWO nicht abzuspielen. Offenbar haben die Qualitätsziele nicht den angenommenen Stellenwert innerhalb der Organisation: Weder bei der Formulierung im Vorjahr, noch beim Monitoring während oder der Evaluation am Ende des Jahres ist die Abteilung Freiwilligendienste größerem Druck seitens der Geschäftsführung ausgesetzt. Herr C. sagt dazu, dass die Vorgaben des Landesverbandes „meistens so allgemein gehalten“ seien und „wir [d. h. die Abteilung Freiwilligendienste] schon wesentlich konkreter werden“. Zur Verdeutlichung der top-down Logik soll ein Qualitätsziel beispielhaft skizziert werden (siehe Tabelle 4). Der Bundesverband hat als thematischen Schwerpunkt das Thema „Nachhaltigkeit“ gesetzt. Der Landesverband formulierte daraufhin in seiner strategischen Ausrichtung für 2014 - 2016 Nachhaltigkeit und Umweltschutz als grundsätzliches Kriterium für alle AWO-Einrichtungen, um die Umweltverträglichkeit zu verbessern und Verantwortung gegenüber der Gesellschaft wahrzunehmen. Als Qualitätsziel für 2016 ist die „Berücksichtigung von Nachhaltigkeit in allen Aufgabenfeldern“ vorgegeben. Die Abteilung Freiwilligendienste hat sich schließlich für konkrete Maßnahmen zur nachhaltigen, ökologischen Weiterentwicklung bis Ende des III. Quartals 2016 entschieden.

Tabelle 4: Ausformulierte Qualitätsziele am Beispiel „Nachhaltigkeit“ (top-down)

\begin{tabular}{|c|c|}
\hline Ebene & Beispiel \\
\hline Bundesverband & Thematischer Schwerpunkt $\rightarrow$ Nachhaltigkeit \\
\hline Landesverband & Vorgabe $\rightarrow$ Berücksichtigung von Nachhaltigkeit in allen Aufgabenfeldern \\
\hline $\begin{array}{l}\text { Abteilung Frei- } \\
\text { willigendienste }\end{array}$ & $\begin{array}{l}\text { Maßnahmen } \rightarrow \text { Der Bereich Freiwilligendienste wird im Rahmen der } \\
\text { nachhaltigen, ökologischen Weiterentwicklung } \\
\text { - die finanziellen Vereinbarungen erstmals online verschicken, } \\
\text { - auf Kopien der Rechnungen zur Einsatzstellenumlagen für den internen } \\
\text { Gebrauch verzichten, } \\
\text { - eine Verknüpfung bezüglich der Einsicht zur Rechnungslegung der Ein- } \\
\text { satzstellenumlage mit der Finanzbuchhaltung erstellen. }\end{array}$ \\
\hline
\end{tabular}

Quelle: Strategische Ausrichtung 2014-16 der AWO Berlin, Qualitätsziele 2016 der Abteilung Freiwilligendienste; eigene Darstellung. 
An einem weiteren Beispiel der Verbesserung des Arbeitsklimas durch einen Teamtag wird die bottom-up Logik dargestellt (siehe Tabelle 5). Dieses Qualitätsziel wurde auf die Interessen und Bedürfnisse der Mitarbeitenden zugeschnitten und autonom vom Landesverband erstellt.

Tabelle 5: Ausformulierte Qualitätsziele am Beispiel „Arbeitsklima“ (bottom-up)

\begin{tabular}{l|l|}
\hline Ebene & Beispiel \\
\hline Bundesverband & Kein thematischer Schwerpunkt \\
\hline Landesverband & Keine Vorgabe \\
\hline $\begin{array}{l}\text { Abteilung Frei- } \\
\text { willigendienste }\end{array}$ & $\begin{array}{l}\text { Der Bereich Freiwilligendienste wird zur weiteren Verbesserung des Ar- } \\
\text { beitsklimas und des vertiefenden, gegenseitigen Kennenlernens der Mit- } \\
\text { arbeiter*innen einen Teamtag organisieren. }\end{array}$ \\
\hline
\end{tabular}

Quelle: Qualitätsziele 2016 der Abteilung Freiwilligendienste; eigene Darstellung.

Das Monitoring der Qualitätsziele im Jahresverlauf ist Bestandteil der Aufgaben des Qualitätszirkels in Verbindung mit der Leitung und der wirtschaftlichen Koordination. Am Ende des Jahres muss der Umsetzungsstand in Prozent zur Beurteilung durch das QM des Landesverbandes vorgelegt werden. Ziele, welche nicht erreicht wurden, werden nicht sanktioniert, sollen aber zeitnah im nächste Jahr umgesetzt werden (Kategorie Qualitätsziele, Unterkategorie Sanktionen). Herr Q. sagt dazu:

„Die Einrichtung bewertet den Umsetzungsstand ihrer Ziele. Dann gucken wir als QM drauf, gehen wir da mit oder haben die geschummelt. Müssen wir da ein Auge zudrücken oder müssen wir da nochmal nacharbeiten."

Im Hinblick auf die Interessendurchsetzung der pädagogischen Mitarbeitenden wird auf die vorangegangenen Ergebnisse bezüglich der allgemeinen Einflussmöglichkeiten im QMS verwiesen. Dort wurde bereits festgestellt, dass es den Mitarbeitenden generell schwerfällt, die vorhandenen Möglichkeiten zu nutzen. Dies ist bei den Qualitätszielen nicht anders. Vor dem Hintergrund, dass sich bei der AWO Berlin scheinbar nicht die Logik der Effizienz durchgesetzt hat, ist für die Mitarbeitenden keine existenzielle Notwendigkeit gegeben ihre Fachlichkeit gegen die in Kapitel 2 beschriebene Ökonomisierung der Sozialen Arbeit bewahren zu müssen. Dementsprechend sind die Antworten auf die Frage nach den Interessen der pädagogischen Mitarbeitenden wenig aussagekräftig und einseitig auf die Prozess- und Dienstleitungsqualität fokussiert. Ihre eigenen Bedürfnisse, z. B. in Bezug auf Strukturqualität als Basis für ein erfolgreiches Arbeiten, wurden während der Interviews nicht thematisiert. Dies scheint jedoch nicht organisationsspezifisch zu sein, sondern ein Merkmal der gesamten Branche:

„Die [Kolleg*innen] müssen fähig sein, Ideen einzubringen. Man möchte nicht glauben, wie schwierig das ist für Sozialarbeiter*innen vernünftige Iden zu formulieren. Die können alles für ihre Klienten, die können nichts für sich.“ (Herr Q.) 
Fallhypothese H2 („Wenn die Einführung von QMS die Machtverhältnisse innerhalb der NPO verändert, entwickelt sich die Formulierung von Qualitätszielen zu einem Machtkampf zwischen den beteiligten Akteuren und ihren divergierenden Interessen.") muss somit abgelehnt werden. Denn trotz Veränderung der formalen Machtverhältnisse innerhalb der AWO Berlin zu potenziell mehr Macht seitens der Mitarbeitenden entwickelt sich die Formulierung von Qualitätszielen nicht zu einem Machtkampf. Das liegt einerseits daran, dass die Interessen der beteiligen Akteure nicht in der angenommenen Intensität voneinander abweichen. Andererseits können die Abteilungen relativ autonom über ihre Qualitätsziele entscheiden und müssen sich nicht einer an Kosten-Nutzen-Denken orientierten Rationalität beugen. Die kooperative Art der Kommunikation und Entscheidungsfindung innerhalb der Organisation wirkt folglich der Entstehung von Machtkämpfen entgegen.

\subsection{Anwendung mikropolitischer Taktiken}

Entgegen der formulierten Erwartung findet bei der Formulierung von Qualitätsziele innerhalb der AWO Berlin kein Machtkampf statt, bei dem sich divergierende Interessen gegeneinander durchsetzen müssen. Die Fallhypothese $\mathrm{H}_{3}$ („Wenn sich die Formulierung von Qualitätszielen als Machtkampf darstellt, dann werden alle Akteure mikropolitische Taktiken nutzen, um ihre Interessen durchzusetzen.") ist somit nichtig und muss abgelehnt werden.

Aus diesem Grund wird an dieser Stelle auf das weitaus häufiger genannte Instrument der Veränderungsmitteilung (auch Verbesserungsvorschlag, insgesamt 22 Nennungen) eingegangen. Da das QMS der AWO wie in Kapitel 2.2 beschrieben, seinen Schwerpunkt auf die Sicherstellung der Prozessqualität legt, ist die Beeinflussung von Verfahrensabläufen und Arbeitsanweisungen von zentraler Bedeutung. QM bedeutet für alle Mitarbeitenden, unabhängig von ihrer Aktivität oder Passivität, die Nutzung von Formularen und Einhaltung von Verfahren. Deshalb ist die Möglichkeit diese zu verändern viel wichtiger als die Erarbeitung strategischer Qualitätsziele. Dort zeigt sich dann zudem ein Verhalten, was vorsichtig als mikropolitische Taktiken - Konsultation mit anschließender Koalitionsbildung sowie Einschmeicheln - gedeutet werden kann.

Größere Veränderungen des QMS müssen Zustimmung im Qualitätszirkel und in der Teamsitzung finden. Um abzuschätzen, ob es sich lohnt den Aufwand der formellen Veränderungsmitteilung zu gehen, ist es sinnvoll bereits zuvor im QMS aktive Mitarbeitende um ihre Meinung zu fragen (Konsultation) und sie von dem eigenen Vorschlag zu überzeugen, damit sie diesen unterstützen (Koalitionsbildung). Wie das funktionieren kann, beschreibt Herr B. an folgendem Beispiel:

„Ich hatte es Anfang dieser Woche, dass ein Kollege auf mich zugekommen ist und meinte, es wäre doch eine tolle Idee, wenn wir die Excel-Tabelle A mit Tabelle B automatisiert verknüpfen könnten, ohne dass wir immer händisch die aktuellen Veränderungen einpflegen müssen. Da habe ich gesagt: Das ist eine Spitzenidee, schreib doch einfach eine Veränderungsmitteilung und lass uns alle daran teilhaben." 
Das zweite Beispiel betrifft ein alternatives Vorgehen bezüglich der Veränderungsmitteilungen für im QMS passive Mitarbeitende (siehe Abbildung 2). Frau A. schildert die Möglichkeit das Schreiben der Veränderungsmitteilungen an andere zu delegieren. Sie bezeichnet diesen Vorgang als „Übersetzung“. Eine zentrale Rolle nimmt der Qualitätsbeauftragte ein, da er als Filter für Veränderungsmitteilungen fungiert. Herr D. sieht sich selbst in einer assistierenden Position für die Mitarbeitenden und erläutert:

„Klammer auf, Klammer zu, nicht fürs Protokoll. Um die QM-Arbeit aufrecht zu erhalten, ich will es mal so sagen, gibt es (...) von mir den Hinweis, dass es mir auch ausreicht, wenn sie mir z. B. das Formblatt (...) rot anmalen und mir ins Fach legen und sagen 'Guck mal, das stimmt nicht'. Und dann mach ich diese Veränderungsmitteilung mit dem Namen, der es genannt hat. (...) Bevor es runterfällt, bevor wir gar keine Information bekommen, dass in so einem Formblatt ein Fehler enthalten ist, inhaltlich oder wie auch immer. Da ist es mir lieber, es gibt diesen kurzen Weg. Dann sitze ich hier und mach das Ding fertig."

Abbildung 2: Formale Struktur und Entkopplung bei der Bearbeitung von Veränderungsmitteilungen in der Abteilung Freiwilligendienste

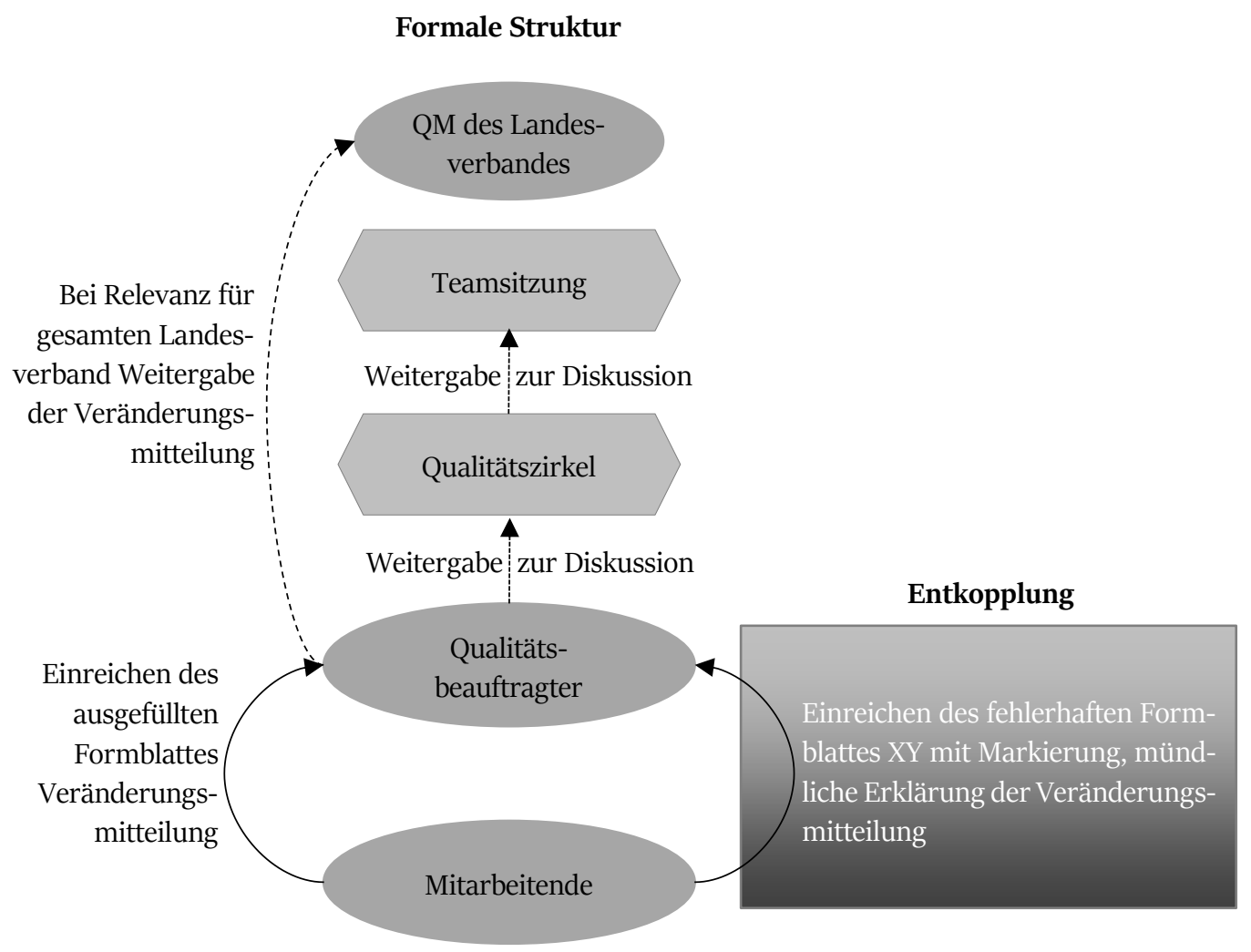

Quelle: Interviewaussagen; eigene Darstellung.

Die Aussagen der pädagogischen Mitarbeitenden Frau A. und des Qualitätsbeauftragten Herr D. sind bezüglichen der alternativen Vorgehensweise konsistent. Die Entkopplung der tatsächlichen Vorgehensweise von den formalen Regeln ermöglicht es für beide Akteure den Vorgang der Verschriftlichung mit weniger Aufwand und höherer Zufriedenheit abzuschließen. Die formale Struktur wird aufrechterhalten und die Mitteilung effizient bearbeitet. 
Allerdings ist es fraglich, inwieweit die beschriebenen Vorgehensweisen bereits als mikropolitische Taktik verstanden werden können. Diese haben zum Ziel, „die Zielperson zum Werkzeug der oft unausgesprochenen, zuweilen getarnten Interessen des Akteurs machen [zu] wollen" (Neuberger 2006, 85). Hier jedoch sind die Interessen keineswegs unausgesprochen oder gar getarnt, sondern es sind Vorgehensweisen, die zur Reduzierung von Transaktionskosten der Beteiligten beitragen. Die im QMS aktiv Mitarbeitenden formulieren erst dann eine Veränderungsmitteilung, wenn sich deren Erfolg durch die Zustimmung der vorab Konsultierten als wahrscheinlicher darstellt. Die im QMS passiven Mitarbeitenden delegieren das Schreiben der Veränderungsmitteilung. Der Qualitätsbeauftragte minimiert seinen Aufwand, indem er sich die Veränderungsmitteilung erklären lässt und gleich in einer adäquaten und für ihn weiter bearbeitbaren Form verschriftlicht. Zudem stellen die genannten Vorgehensweisen keine vorausgeplanten Handlungsmuster zur Verfolgung einer langfristigen Strategie dar, sondern entstehen situativ und kommen nur gelegentlich vor.

\section{Fazit}

Die Ergebnisse der Fallstudie sind mit dem in Kapitel 3 dargestellten Forschungsstand konsistent. Alle befragten Mitarbeitenden haben die Verbesserung der internen Arbeitsabläufe und das organisationale Lernen erwähnt und bei einigen Mitarbeitenden führte QM zu einer Motivationssteigerung (Vgl. Cairns u. a. 2005; Melão und Guia 2015; Melão, Guia, und Amorim 2016), diese wurden als aktive Mitarbeitende bezeichnet. Jedoch sind die Hürden, die Sozialarbeiter*innen bewältigen müssen, um ihre Interessen im QMS aktiv durchzusetzen, recht hoch. Erst nach erfolgreicher Wissensaneignung um die Spielregeln von QM und bei gleichzeitigem Vorhandensein zeitlicher Ressourcen können sie als mikropolitische Spieler*innen den Machtkampf in der organisatorischen Arena bestreiten. Innerhalb der AWO Berlin führt das QM zu mehr Einflussmöglichkeiten seitens der Mitarbeitenden im Rahmen der strategischen Organisationsentwicklung, die bisher jedoch weitestgehend ungenutzt bleiben. Ihr derzeitiger Einfluss auf die Formulierung von Qualitätszielen ist sehr heterogen. Einen großen Einfluss haben die im QMS aktiven und im Qualitätszirkel involvierten Mitarbeitenden: Sie passen die Themenvorgaben des Bundes- und Landesverbandes an die Gegebenheiten und Bedürfnisse vor Ort an (top-down) oder formulieren abteilungsspezifische Qualitätsziele (bottom-up). Einen geringen Einfluss haben die im QMS passiven Mitarbeitenden. Für deren Arbeitsalltag spielen Qualitätsziele aber auch keine hinreichend bedeutende Rolle, als dass sie diesen Umstand als Mangel wahrnehmen. Es bestehen wenig bis kaum divergierenden Interessen zwischen den verschiedenen Akteur(sgruppen), die die Entstehung von Machtkämpfen begünstigen würden. Darüber hinaus hemmt die außerordentlich kooperative Art der Kommunikation und Entscheidungsfindung etwaige Tendenzen. Diese Falleigenschaften erklären, wieso der mikropolitische Ansatz bei der Analyse der organisationsinternen Prozesse nicht zu den antizipierten Resultaten geführt hat.

Doch solange die Mitarbeitenden ihre Einflussmöglichkeiten ungenutzt lassen, bleibt QM ein Instrument zur Kontrolle durch die Leitung. In der Sozialen Arbeit, deren Ziel eine individuelle und 
alltagsorientierte Ausrichtung (sozial-)pädagogischen Handelns ist (Merchel 2004, 139), scheint das kein angemessenes Managementkonzept zu sein. Vielmehr müssen die pädagogischen Mitarbeitenden befähigt werden, relevante Themen aus ihrer alltäglichen Arbeit oder auf Basis von aktuellen, gesellschaftlichen Herausforderungen in die strategische Organisationsentwicklung einfließen zu lassen. Zugleich müssen NPOs ihren Fachkräften zugestehen, dass soziale Dienstleistungen nicht nach QM-Kriterien funktionieren, die von Industrieunternehmen erstellt und daraufhin auf die Bedürfnisse von NPOs zugeschnitten wurden. Erst in einem zweiten Schritt können sich die positiven Wirkungen entfalten. Zudem ist es aus Sicht der Organisation hinderlich, wenn QM zu einem Faktor wird, der zu einer Frontenbildung aus engagierten, leidenschaftlichen und gleichgültig, ablehnenden Kolleg*innen führt, anstatt die Ideen und Vorstellungen aller als Veränderungspotentiale zu nutzen. Das trifft insbesondere für vergleichbare Organisationen wie dem Fallbeispiel der AWO Berlin zu, welche eine sehr kooperative Umgangsweise miteinander pflegt und den einzelnen Einheiten eine hohe Autonomie zugesteht.

Die zukünftige Forschung sollte sich verstärkt mit der „Übersetzung“ und Anpassung von betriebswirtschaftlichen Konzepten an die Eigenschaften anderer Organisationsformen auseinanderzusetzen. Die Kluft zwischen Theorie und Praxis ist auch zwanzig Jahre nach Start der Qualitätsdebatte in der Sozialen Arbeit zu groß. Es scheint, als ob die Idee des Einbezugs der Mitarbeitenden zwar intendiert wird, aber nicht erfolgreich umgesetzt wird. Zur vertiefenden Analyse von mikropolitischen Effekten durch QM wäre eine vergleichende, empirische Betrachtung anderer Organisationen aus dem Nonprofit-Sektor oder anderen Sektoren interessant. Es wäre zu untersuchen, ob die in dieser Arbeit durchgeführte Fallstudie lediglich eine besonders regelkonforme und kooperative Organisation abbildet und ob in anderen Organisationen durchaus zwischen Akteuren mit divergierenden Interessen Machtkämpfe um Qualitätsziele mithilfe von mikropolitischen Taktiken und Strategien in der organisatorischen Arena stattfinden. 


\section{Literaturverzeichnis}

Arnold, Ulli. 2014 ."Qualitätsmanagement in Sozialwirtschaftlichen Organisationen“. In Lehrbuch der Sozialwirtschaft, herausgegeben von Ulli Arnold, Klaus Grunwald, und Bernd Maelicke, 4., erweiterte Auflage, 585-628. Baden-Baden: Nomos.

Atteslander, Peter. 2008. Methoden der empirischen Sozialforschung. 12. Auflage. Berlin: Erich Schmidt Verlag.

Beckmann, Christof, Hans-Uwe Otto, Martina Richter, und Mark Schrödter. 2004. „Negotiating Qualities - Ist Qualität eine Verhandlungssache?" In Qualität in der Sozialen Arbeit, herausgegeben von Christof Beckmann, Hans-Uwe Otto, Martina Richter, und Mark Schrödter, 931. Wiesbaden: VS Verlag für Sozialwissenschaften.

Blickle, Gerhard. 2004. „Einflusskompetenz in Organisationen“. Psychologische Rundschau 55 (2): 82-93.

Bogumil, Jörg, und Josef Schmid. 2001. Politik in Organisationen: organisationstheoretische Ansätze und praxisbezogene Anwendungsbeispiele. Grundwissen Politik 31. Opladen: Leske + Budrich.

Bruhn, Manfred. 2013. Qualitätsmanagement für Nonprofit-Organisationen. Wiesbaden: Springer Gabler.

Brüsemeister, Thomas. 2008. Qualitative Forschung: ein Überblick. 2., überarbeitete Auflage. Wiesbaden: VS Verlag für Sozialwissenschaften.

Bumbacher, Urs. 1998. „10 Thesen über Qualität und Qualitätsmanagement in Nonprofit-Organisationen (NPO)“. Zeitschrift für öffentliche und gemeinwirtschaftliche Unternehmen 21 (3): $342-49$.

Butterwegge, Christoph, Martin Kutscha, und Sabine Berghahn. 1999. Herrschaft des Marktes, Abschied vom Staat?: Folgen neoliberaler Modernisierung für Gesellschaft, Recht und Politik. Baden-Baden: Nomos.

Cairns, Ben, Margaret Harris, Romayne Hutchison, und Mike Tricker. 2005. „Improving Performance? The Adoption and Implementation of Quality Systems in UK Nonprofits“. Nonprofit Management and Leadership 16 (2): 135-51.

Crozier, Michel, und Erhard Friedberg. 1979. Macht und Organisation: die Zwänge kollektiven Handelns. Königstein/Taunus: Athenäum.

Dahme, Heinz-Jürgen, Gertrud Kühnlein, Norbert Wohlfahrt, und Monika Burmester. 2008. Zwischen Wettbewerb und Subsidiarität: Wohlfahrtsverbände unterwegs in die Sozialwirtschaft. 2. Auflage. Berlin: edition sigma.

DIN EN ISO 9000:2005. 2005. Qualitätsmanagementsysteme - Grundlagen und Begriffe. Berlin.

Göbel, Markus. 1999. Verwaltungsmanagement unter Veränderungsdruck. München: Rainer 
Hampp Verlag.

Grohs, Stephan. 2010. „Lokale Wohlfahrtsarrangements zwischen Behaarung und Wandel: Die widersprüchlichen Effekte von Ökonomisierung und Kontraktmanagement". der moderne staat - Zeitschrift für Public Policy, Recht und Management 3 (2): 413-32.

Hopf, Christel. 2016. „Hypothesenprüfung und qualitative Sozialforschung“. In Schriften zu Methodologie und Methoden qualitativer Sozialforschung, von Christel Hopf, 155-66. Wiesbaden: Springer Fachmedien Wiesbaden.

Jones, Jeri L., und Mahmood Shandiz. 2015. „Service Quality Expectations: Exploring the Importance of SERVQUAL Dimensions from Different Nonprofit Constituent Groups“. Journal of Nonprofit \& Public Sector Marketing 27 (1): 48-69.

Kennedy, Mark Thomas, und Peer Christian Fiss. 2009. „Institutionalization, Framing, and Diffusion: The logic of TQM adoption and implementation among U.S. hospitals". Academy of Management Journal 52 (5): 897-918.

Kühl, Stefan. 2001. „Paradoxe Effekte und ungewollte Nebenfolgen des Qualitätsmanagements“. In Qualitätsmanagement in Organisationen: DIN ISO gooo und TQM auf dem Prüfstand, herausgegeben von Hartmut Wächter und Günther Vedder, 75-113. Wiesbaden: Springer Gabler.

Kulbach, Roderich, und Norbert Wohlfahrt. 1996. Modernisierung der öffentlichen Verwaltung? Konsequenzen für die freie Wohlfahrtspflege. Freiburg im Breisgau: Lambertus-Verlag.

Liao, Ying-Ying, Ebrahim Soltani, und Pamela Yeow. 2014. „What Sparks Quality-Driven Change Programmes in Not-for-Profit Service Sector? Some Evidence from the Voluntary Sector". Total Quality Management \& Business Excellence 25 (11-12): 1295-1317.

Mayring, Philipp. 2008. Einführung in die qualititative Sozialforschung: eine Anleitung zu qualitativem Denken. 5. Auflage. Weinheim Basel: Beltz.

- - . 2010. Qualitative Inhaltsanalyse: Grundlagen und Techniken. 11., aktualisierte und überarbeitete Auflage. Weinheim: Beltz.

Melão, Nuno Filipe, und Sara Maria Guia. 2015. „Exploring the Impacts of ISO 9001 on Small- and Medium-Sized Social Service Institutions: A Multiple Case Study“. Total Quality Management \& Business Excellence 26 (3-4): 312-26.

Melão, Nuno Filipe, Sara Maria Guia, und Marlene Amorim. 2016. „Quality Management and Excellence in the Third Sector: Examining European Quality in Social Services (EQUASS) in NonProfit Social Services“. Total Quality Management \& Business Excellence, 1-18.

Merchel, Joachim. 2004. „Qualität als Verhandlungssache“. In Qualität in der Sozialen Arbeit, herausgegeben von Christof Beckmann, Hans-Uwe Otto, Martina Richter, und Mark Schrödter, 133-54. VS Verlag für Sozialwissenschaften.

Moldaschl, Manfred. 2001. „Qualität als Spielfeld und Arena: Das mikropolitische Verständnis von 
Qualitätsmanagement - und seine Grenzen“. In Qualitätsmanagement in Organisationen: DIN ISO gooo und TQM auf dem Prüfstand, herausgegeben von Hartmut Wächter und Günther Vedder, 115-38. Wiesbaden: Springer Gabler.

Neuberger, Oswald. 1995. Mikropolitik: der alltägliche Aufbau und Einsatz von Macht in Organisationen. Stuttgart: Enke.

-- - 2006. Mikropolitik und Moral in Organisationen: Herausforderung der Ordnung. 2., völlig neu bearbeitete Auflage. Stuttgart: Lucius \& Lucius.

Powell, Thomas C. 1995. „Total Quality Management as Competitive Advantage: A Review and Empirical Study“. Strategic Management Journal 16 (1): 15-37.

Schnell, Rainer, Paul B. Hill, und Elke Esser. 2005. Methoden der empirischen Sozialforschung. 7., völlig überarbeitete und erweiterte Auflage. München: Oldenbourg.

Soltani, Ebrahim, Pei-Chun Lai, und Vahid Mahmoudi. 2007. „Managing Change Initiatives: Fantasy or Reality? The Case of Public Sector Organisations“. Total Quality Management \& Business Excellence 18 (1-2): 153-79.

Süß, Stefan. 2009. „Die Institutionalisierung von Managementkonzepten. Eine strukturationstheoretisch-mikropolitische Perspektive“. Zeitschrift für Betriebswirtschaft 79 (2): 187-212.

Tolbert, Pamela S., und Lynne G. Zucker. 1983. „Institutional Sources of Change in the Formal Structure of Organizations: The Diffusion of Civil Service Reform, 1880-1935“. Adminstrative Science Quarterly 28 (1): 22-39.

Waldman, David A., Terri Lituchy, Mohan Gopalakrishan, Kevin Laframboise, Bella Galperin, und Zefi Kaltsounakis. 1998. „A Qualitative Analysis of Leadership and Quality Improvement“. Leadership Quarterly 9 (2): 177-201.

Walgenbach, Peter, und Renate E. Meyer. 2008. Neoinstitutionalistische Organisationstheorie. Stuttgart: Kohlhammer.

Westphal, James D., Ranjay Gulati, und Stephen M. Shortell. 1997. „Customization or Conformity? An Institutional and Network Perspective on the Content and Consequences of TQM Adoption“. Adminstrative Science Quarterly 42 (2): 366-94. 


\section{Schriftenreihe für Public und Nonprofit Management}

Herausgegeben vom Lehrstuhl für Public und Nonprofit Management der Wirtschafts- und Sozialwissenschaftlichen Fakultät der Universität Potsdam

\section{In dieser Reihe erschienen:}

Band 1 Polzer, Tobias: Verwendung von Performance-Informationen in der öffentlichen Verwaltung : eine Untersuchung der Berliner Sozialhilfeverwaltung / Tobias Polzer. - $83 \mathrm{~S}$. 2010 | URN urn:nbn:de:kobv:517-opus-42357

Band 2 Radke, Marlen: Die Balanced Scorecard zur Unterstützung der politischen Planung und Steuerung der Vorhaben einer Landesregierung / Marlen Radke. - $85 \mathrm{~S}$.

2010 | URN urn:nbn:de:kobv:517-opus-42395

Band 3 Krischok, Arndt: Die Rolle von Policy-Netzwerken in Public Private Partnerships / Arndt Krischok. - $98 \mathrm{~S}$.

2010 | URN urn:nbn:de:kobv:517-opus-43046

Band $4 \quad$ Vogel, Dominik: Dem Gemeinwohl verpflichtet? - Was motiviert die Beschäftigten des öffentlichen Dienstes? / Dominik Vogel. - $75 \mathrm{~S}$. 2011 | URN urn:nbn:de:kobv:517-opus-51554

Band 5

Rackow, Maja: Personalmarketing in der öffentlichen Verwaltung : Analyse und Implikationen eines Best Practice-Ansatzes /

Maja Rackow. - VI, $68 \mathrm{~S}$.

2011 | URN urn:nbn:de:kobv:517-opus-51652

Band 6 Schnitger, Moritz: Pflegekonferenzen als geeignetes Instrument zur Optimierung des deutschen Pflegemarktes? : Steuerungspotential lokaler Politiknetzwerke im Rahmen von Wohlfahrtsmärkten /

Moritz Schnitger. - VI, $137 \mathrm{~S}$.

2011 | URN urn:nbn:de:kobv:517-opus-52567

Band 7 Kunath, Marcus: Personalpolitik in der Landesverwaltung und demografischer Wandel : unausgewogene Altersstrukturen als Handlungsfeld des strategischen Personalmanagements in den Landesverwaltungen Berlin und Hamburg /

Marcus Kunath. - vi, $93 \mathrm{~S}$.

2011 | URN urn:nbn:de:kobv:517-opus-53386

\section{Band 8}

Hengel, Martin: Beteiligungsmanagement in Zeiten des kommunalen Gesamtabschlusses / Martin Hengel. - iii, 67 S. 
Band 9

Band 10

Band 11

Band 12

Band 13

Band 14

Band 15

Band 16

Band 17
Nijaki, Nadine: Public Service Motivation im Nonprofit-Bereich : eine Fallstudie am Beispiel des Deutschen Roten Kreuzes / Nadine Nijaki. - 26, XVI S.

2011 | URN urn:nbn:de:kobv:517-opus-54487

Was machen Verwaltungsmanager wirklich? : Explorative Ergebnisse eines Lehrforschungsprojekts / Alexander Kroll, John Philipp Siegel (Hrsg.). - 66 S. 2011 | URN urn:nbn:de:kobv:517-opus-54526

Kramer, Ansgar: Organisationale Fähigkeiten des öffentlichen Sektors : zur Übertragbarkeit der Capability Based View auf die Öffentliche Verwaltung / Ansgar Kramer. - $68 \mathrm{~S}$.

2012 | URN urn:nbn:de:kobv:517-opus- 57298

Döring, Matthias: Der Einfluss von Verwaltungskultur auf die Verwendung von Performance-Daten : eine quantitative Untersuchung der deutschen kreisfreien Städte / Matthias Döring. - $28 \mathrm{~S}$.

2012 | URN urn:nbn:de:kobv:517-opus-57698

Bögel, Simon: Anreize bei der Budgetierung : Welche dysfunktionalen Verhaltensweisen der Manager resultieren aus der Berliner MedianBudgetierung? / Simon Bögel. - VI, 66 S.

2012 | URN urn:nbn:de:kobv:517-opus-58124

Faasch, Britta: Der Einfluss der leistungsorientierten Bezahlung auf die Public Service Motivation und die intrinsische Motivation von Beschäftigten im öffentlichen Sektor: Ein empirischer Test der Motivation Crowding Theory am Beispiel der Kreisverwaltung Potsdam-Mittelmark/ Britta Faasch. - VI, $73 \mathrm{~S}$.

2012 | URN urn:nbn:de:kobv:517-opus-61892

Kalm, Nicolas von: Personalführung in der öffentlichen Verwaltung in Zeiten des demographischen Wandels : eine Untersuchung der Wirkung altersspezifischer Führung auf die Arbeitsbeziehung von Führungskraft und Mitarbeiter am Beispiel einer Dienststelle der Bundesagentur für Arbeit / Nicolas von Kalm. - VI, $66 \mathrm{~S}$.

2012 | URN urn:nbn:de:kobv:517-opus-63056

Wenzek, Eva: Organisationale Fähigkeiten in Museen :

eine explorative Fallstudie / Eva Wenzek. - XVI, $28 \mathrm{~S}$.

2012 | URN urn:nbn:de:kobv:517-opus- 63645

Muriu, Abraham Rugo: Decentralization, citizen participation and local public service delivery : a study on the nature and influence of citizen 
participation on decentralized service delivery in Kenya / Abraham Rugo Muriu. - VIII, $79 \mathrm{~S}$.

2013 | URN urn:nbn:de:kobv:517-opus-65085

Band 18 Nickenig, Julia: Mitarbeitermotivation in der Wissenschaft am Beispiel des Leibniz-Instituts für Agrartechnik Potsdam-Bornim e. V. / Julia Nickenig. - vi, $76 \mathrm{~S}$.

2014 | URN urn:nbn:de:kobv:517-opus-71353

Band 19 Creusen, Leander: Die Arbeit der Organisationseinheit „Beteiligungsmanagement“ im Gesamtkontext der Steuerung öffentlicher Unternehmen auf kommunaler Ebene / Leander Creusen. - VI, $82 \mathrm{~S}$.

2014 | URN urn:nbn:de:kobv:517-opus- 71938

Band 20 Ansel, Simon: Die Diffusion von Innovationen in deutschen Kommunen : eine Untersuchung zu Komponenten des E-Government / Simon Ansel - IV, $67 \mathrm{~S}$.

2015 | URN urn:nbn:de:kobv:517-opus4- 80370

Band 21 Schinck, Kai Philipp: Erfolgsfaktor Qualitätsmanagement? Die effektive Implementierung von Qualitätsmanagementsystemen in öffentlichen Organisationen / Kai Philipp Schinck - 54 S.

2017 | URN urn:nbn:de:kobv:517-opus4-400520

Band 22 Marienfeldt, Justine: Qualitätsmanagement in Nonprofit-Organisationen : eine mikropolitische Analyse am Beispiel des Landesverbandes der Arbeiterwohlfahrt Berlin / Justine Marienfeldt - I, $30 \mathrm{~S}$.

2018 | URN urn:nbn:de:kobv:517-opus4- 408877 
